\title{
Hepatocyte-specific suppression of microRNA-221-3p mitigates liver
}

\section{fibrosis}

Hsin-Chieh Tsay ${ }^{1,2,3^{*}}$, Qinggong Yuan ${ }^{2,3^{*}}$, Asha Balakrishnan ${ }^{2,3}$, Marina

Kaiser $^{4}$, Selina Möbus ${ }^{1,2}$, Emilia Kozdrowska ${ }^{1,2}$, Marwa Farid ${ }^{1,2,3,5}$,

Pia-Katharina Tegtmeyer ${ }^{3,6}$, Katharina Borst ${ }^{3,6}$, Florian W.R. Vondran ${ }^{7,8}$, Ulrich

Kalinke $^{3,6}$, Andreas Kispert ${ }^{4}$, Michael P. Manns ${ }^{2}$, Michael Ott ${ }^{2,3}$, Amar Deep

Sharma $^{1,2}$

${ }^{1}$ Research Group MicroRNA in Liver Regeneration, Cluster of Excellence REBIRTH, Hannover Medical School, Hannover, Germany

${ }^{2}$ Department of Gastroenterology, Hepatology and Endocrinology, Hannover Medical School, Hannover, Germany

${ }^{3}$ TWINCORE, Centre for Experimental and Clinical Infection Research, Hannover, Germany

${ }^{4}$ Institute for Molecular Biology, Hannover Medical School, Hannover, Germany

${ }^{5}$ Human Cytogenetics Department, Human Genetics and Genome Research Division, National Research Centre, Cairo, Egypt

${ }^{6}$ Institute for Experimental Infection Research, TWINCORE, Centre for Experimental and Clinical Infection Research, a joint venture between the 
Helmholtz Centre for Infection Research and the Hannover Medical School,

Hannover, Germany

${ }^{7}$ Regenerative Medicine and Experimental Surgery (RedMediES), Department of General, Visceral and Transplant Surgery, Hannover Medical School, Hannover, Germany.

${ }^{8}$ German Centre for Infection Research (DZIF), partner site Hannover-Braunschweig, Hannover, Germany.

${ }^{*}$ These authors share first authorship.

\section{Corresponding authors:}

Dr. Amar Deep Sharma, PhD

Hannover Medical School

Carl-Neuberg Str. 1, Hannover 30625, Germany

Phone: 0049-5115325255, Fax: 0049-5115325234

Email: sharma.amar@mh-hannover.de

And

Prof. Michael Ott, PhD

Hannover Medical School

Carl-Neuberg Str. 1, Hannover 30625, Germany 
Phone: 0049-5115327120, Fax: 0049-5115327186

Email: ott.michael@mh-hannover.de 


\section{Abstract}

Background \& Aims: Fibrosis, a cardinal feature of a dysfunctional liver, significantly contributes to the ever-increasing mortality due to end-stage chronic liver diseases. The cross-talk, between hepatocytes and hepatic stellate cells, is suggested to play a key role in fibrosis progression. While ample efforts have been devoted to elucidate hepatic stellate cells' functions during liver fibrosis, the regulatory functions of hepatocytes remain elusive. Methods: Using an unbiased functional microRNA screening, we investigated the ability of hepatocytes to regulate fibrosis by fine-tuning gene expression via microRNA modulation. The in vivo functional analyses were performed by inhibiting microRNA in hepatocytes using adeno-associated virus in carbon-tetrachloride- and 3,5-di diethoxycarbonyl-1,4-dihydrocollidine-induced liver fibrosis.

Results: We discovered that blocking microRNA-221-3p function in hepatocytes during chronic liver injury facilitates recovery of the liver and faster resolution of the deposited extracellular matrix. Further, we demonstrate that reduced secretion of $\mathrm{C}-\mathrm{C}$ motif chemokine ligand 2 , due to post-transcriptional regulation of $\mathrm{G}$ protein alpha inhibiting activity polypeptide 2 by microRNA-221-3p, mitigates liver fibrosis. 
Conclusions: Collectively, microRNA modulation in hepatocytes, an easy-to-target cell type in the liver, may serve as a potential therapeutic approach for liver fibrosis.

\section{Lay Summary}

Liver fibrosis majorly contributes to mortality due to various liver diseases. Here, we discovered a small RNA known as microRNA-221-3p, whose downregulation in hepatocytes results in reduced liver fibrosis. Thus, inhibition of microRNA-221-3p may serve as one of the therapeutic approaches for treatment of liver fibrosis. 


\section{Introduction}

Liver fibrosis and cirrhosis contribute to more than 1 million deaths per year worldwide ${ }^{1,2}, 170,000$ per year in Europe ${ }^{3}$ and 33,539 per year in USA ${ }^{4}$. The underlying pathologies leading to fibrosis and subsequently cirrhosis include chronic virus infection, alcoholic steatohepatitis, and non-alcoholic steatohepatitis ${ }^{5}$. The manifestation of fibrosis is accompanied by the activation of quiescent hepatic stellate cells (HSCs), accumulation of excessive extra-cellular matrix and hepatocyte dysfunction leading to liver failure ${ }^{6}$. While the majority of efforts have been concentrated on the elucidation of HSCs function during fibrosis, the regulatory functions of hepatocyte, the main parenchymal cells of liver, remain to be understood further. Specifically, how gene expression alterations, particularly at the post-transcriptional level in hepatocytes, regulate fibrosis remains to be investigated. MicroRNAs (miRNAs), one of the post-transcriptional regulators of gene expression ${ }^{7,8}$, have been reported to be deregulated in liver fibrosis ${ }^{7,9}$. The majority of studies have examined miRNAs' functions in HSCs, however, identification and functional analysis of hepatocyte miRNAs, which are capable of regulating fibrosis, remain to be investigated. Elucidation of such key fibrosis-regulating miRNAs in hepatocytes, an easy-to-target cell type in the liver, would allow 
development of successful therapeutics for liver fibrosis.

\section{Materials and methods}

Animals

8-12 week-old male BALAB/c mice (Charles River) were used for all in vivo studies. Mice (five in one cage) were provided free access to food and water and kept in colony room on a 12:12 hour light and dark cycle. All experiments, within this study, were performed according to animal protocol (TVA 17/2658), which was approved by Hannover Medical School and authorities of Lower Saxony State, Germany.

MiRNA mimic library screening

The 96-well inserts (Corning 3392) were coated with $50 \mu \mathrm{g} / \mathrm{ml}$ collagen (Roche) at room temperature for one hour. After discarding the collagen solution, 6500 primary mouse hepatocytes were seeded to each air-dried insert and were cultured with Hepatocyte Culture Medium (HCM) over night. Total 302 miRNAs which are conserved between human and mouse were applied for the transfection in a final concentration of $25 \mathrm{nM}$ miRNA per well. At 16 hours of post-transfection, the transfected primary mouse hepatocytes were placed on 
top of a monolayer of mHSC-Col1a1-GFP cells (7000 cells per well) in DMEM medium supplemented with 10\% heat-inactivated FBS, 4 mM L-Glutamine, and $1 \%$ Penicillin/Streptomycin for further incubation at $37^{\circ} \mathrm{C}$. The GFP intensity of mHSC-col1a1-GFP cells was measured after 48 hours of co-culture.

Mouse model of liver fibrosis

Animal experiments were performed according to the guidelines of the Institutional Animal Care and Use Committee of the Hannover Medical School, Germany. BALB/c mice (8-12 weeks old) were purchased from the central animal facility of the Hannover Medical School, Germany. To establish liver fibrosis in vivo, BALB/c mice were injected with $4 \mu \mathrm{l}$ of $10 \% \mathrm{CCl}_{4}$ - olive oil (Sigma) per gram of mouse body weight, two times a week for eight weeks. Mice in the control group were injected with only olive oil. In situ hybridization analysis In situ hybridization analysis was performed on $4 \mu \mathrm{m}$ paraffin sections following a standard procedure with digoxigenin-labeled antisense riboprobes 10-12 with following exceptions: hybridization occurred at $54^{\circ} \mathrm{C}$ and washing in $50 \%$ formamide $/ 2 \mathrm{x}$ SSC at $50^{\circ} \mathrm{C}$. The final concentration of the miR-221-3p-probe (Exiqon) was $40 \mathrm{nM}$. As a control we used $0.2 \mathrm{nM}$ 
U6-probe.

AAV8 virus production

293T cells, with 60\% confluency, were transfected with $25 \mu \mathrm{g}$ pDP8.ape (Plasmid Factory, Germany) and $25 \mu \mathrm{g}$ pAAV-Ttr-control using the calcium phosphate method. To construct pAAV-Ttr-TuD, we synthesized TuD-miR-221-3p ${ }^{13}$ with restriction sites Sacll and Notl and ligated this into a pAAV-Ttr plasmid. AAV8 virus was produced as previously described ${ }^{14,15}$. Viral titer was determined by qPCR using primers targeting the Ttr promoter.

Primary HSC isolation

The procedure was performed as described ${ }^{16}$. Briefly, five to ten mouse livers from mice with an age minimum of 35 weeks were perfused with Pronase $E$ solution and Collagenase $P$ solution at a flow speed of $6.5 \mathrm{ml} / \mathrm{min}$ for 4.5 minutes at $37^{\circ} \mathrm{C}$. All perfused mouse livers were collected and digested in vitro with $50 \mathrm{ml}$ Pronase $\mathrm{E}$ - Collagenase $\mathrm{P}$ solution and $1 \mathrm{ml} \mathrm{DNasel}$ at $37^{\circ} \mathrm{C}$ for 20 minutes. The cell suspension was filtered through $100 \mu \mathrm{m}$ cell strainers and centrifuged at $600 \mathrm{xg}$ at $4^{\circ} \mathrm{C}$ for 10 minutes. After discarding the supernatant, the cells were resuspended in GBSS-B and $150 \mu$ l DNasel solution was added to the cell suspension. The cell suspension was centrifuged at $600 \mathrm{xg}$ at $4^{\circ} \mathrm{C}$ for 10 minutes. After carefully discarding the supernatant, the cells were 
suspended with $36 \mathrm{ml}$ GBSS-B buffer and $150 \mu$ LNasel solution was added to the solution. The $14 \mathrm{ml}$ Nycodenz solution $(8 \mathrm{~g}$ Nycodenz with $28 \mathrm{ml}$ GBSS-A buffer) was added to the cell suspension to reach a $8 \%$ Nycodenz gradient. The gradients were centrifuged at $1500 \times \mathrm{xg}$ at $4^{\circ} \mathrm{C}$ for 15 minutes without brake. Primary HSCs were harvested from the interphase of the gradients. Primary human myofibroblasts were purchased from Innoprot, Spain. Primary HSCs were cultured with DMEM supplemented with $10 \%$ heat-inactivated FBS, 1\% Penicillin-Streptomycin, and 4 mM L-Glutamine.

Primary hepatocyte transfection

One million primary mouse hepatocytes were seeded per well of collagen-coated 6-well plates. Four hours after seeding, the medium was changed to fresh HCM. Virofect-enhanced hepatocytes were transfected with $100 \mathrm{nM}$ miRNA mimics or scramble control with TargeFect F-2 (Targeting system). The medium was changed to fresh $\mathrm{HCM}$ eight hours post transfection. Cell pellets were collected 48 hours after transfection. Primary human hepatocytes were isolated from liver tissue obtained from patients undergoing partial hepatectomy and according to written informed consent approved by the ethics commission of Hannover Medical School (Ethics-commission of Hannover Medical School, \#252-2008), as previously reported ${ }^{17}$. Primary 
human hepatocytes from fibrotic livers and control (non-fibrotic livers) were purchased from PRIMACYT GmbH.

Co-culture of primary mouse hepatocytes and primary mouse HSCs

100,000 primary hepatocytes were seeded per transwell insert (Corning, 3494) for overnight culture. Hepatocytes were then transfected with either $1 \mu \mathrm{g}$ control vector (pCMV-GFP) or $1 \mu \mathrm{g}$ GNAI2 expression vector (pCMV-GNAI2-GFP, Biocat). After 12 to 16 hours of transfection, the insert was placed on the top of primary mouse HSCs $(200,000$ cells per well) for further 48 hours co-culture. The primary moue HSCs were fixed with 4\% PFA or the cell pellets were collected for further experiments.

FACS analyses for CD11b and F4/80

Mononuclear cell isolation from the liver was performed as previously described ${ }^{16,18}$. After in situ perfusion and in vitro digestion of the liver, cells were filtered through $70 \mu \mathrm{m}$ cell strainers and centrifuged at $300 \mathrm{~g}$ for $8 \mathrm{~min}$ at $4{ }^{\circ} \mathrm{C}$. The cell pellet was resuspended in $33 \%$ Percoll and centrifuged at $500 \mathrm{~g}$ for $15 \mathrm{~min}$ at room temperature. Following red blood cell lysis (Sigma) cells were immunolabeled with fluorochrome-conjugated antibodies (Biolegend and BD) for flow-cytometry analysis (LSR II Sorb, Becton Dickinson). 
Additional methods are provided in the supplementary materials and methods

\section{Statistical Analysis}

Significance was determined with the two-tailed Student's t-test for comparison of two groups. Significance between multiple groups was determined by one-way ANOVA. $P<0.05$ was considered as significant $\left({ }^{*} \mathrm{P}<0.05 ;{ }^{*} \mathrm{P}<0.01\right.$; $\left.{ }^{* * *} \mathrm{P}<0.001\right)$. Error bars represent \pm SEM.

\section{Results}

\section{Functional miRNA screening identifies miR-221-3p as a potential fibrosis} regulator

To identify miRNAs whose modulation in primary hepatocytes is capable of regulating liver fibrosis, we undertook a miRNA screening approach, wherein we examined a fibrogenic role of 302 miRNAs. These miRNAs represent a library of conserved miRNAs between mouse and human. Our miRNA screening required use of a hepatic stellate cell line, which allows measurement of fibrosis-related markers in a high-throughput manner. For this purpose, we used mouse hepatic stellate cells, which express GFP under the transcriptional control of collagen 1 promoter/enhancer (henceforth referred to 
as Col-GFP HSCs) ${ }^{19}$. Thus, we first transfected primary mouse hepatocytes with individual miRNAs and subsequently co-cultured them with Col-GFP HSCs (Fig. 1A). After 48 hours, we measured GFP intensity as surrogate readout of fibrogenesis (Fig. 1B). The GFP intensity of co-cultured cells transfected with scramble control (Scr) was set as 1. Based on this screening, we selected 15 miRNAs, which demonstrated maximum elevation in GFP intensity. We then validated those 15 miRNAs in three independent co-culture experiments. 12 out of 15 miRNAs showed similar results compared to those obtained from our miRNA screen. Notably, 6 out of these 12 miRNAs significantly $(P<0.05)$ increased GFP intensity (Fig. 1C) indicating these 6 miRNAs may promote fibrogenesis. These 6 miRNAs were miR-151a-5p, $-374 b-5 p,-200 b-3 p,-219-2-3 p,-221-3 p$ and $-30 c-1-3 p$. In addition, we also found two miRNAs (miR-17-5p and miR-7-1-3p), whose transfection in primary hepatocytes reduced GFP intensity (Fig. 1C) indicating these two miRNAs may serve as negative regulator of fibrosis.

Tissue inhibitor of metalloproteinases 1 (TIMP1) is secreted by activated HSCs and inhibits metalloproteinases to suppress extracellular matrix degradation ${ }^{20,21}$. We therefore measured levels of secreted TIMP1, as an unbiased second parameter to identify miRNAs, which are capable of 
regulating fibrosis. Based on three independent experiments, we found that transfection of miR-221-3p and miR-17-5p mimics led to significant increase in TIMP1 levels as shown by ELISA (Fig. 1D and Supplementary Fig. S1A). We then confirmed elevation in TIMP1 levels in primary mouse HSCs (instead of Col-GFP HSCs) that were co-cultured with primary hepatocytes transfected either with miR-221-3p (Fig. 1E) or miR-17-5p mimic (Supplementary Fig. S1B). The transfection of miR-221-3p (Fig. 1E) but not miR-17-5p mimics (Supplementary Fig. S1B) led to significant elevation in TIMP1 levels, indicating that only gain of miR-221-3p in hepatocytes affected fibrogenesis from co-cultured primary mouse HSCs. Furthermore, miR-221-3p mimic transfection also led to higher mRNA levels of characteristic fibrogenic markers such as Col1a1, Acta2 and Tgfbr1 (Fig. 1E). To rule out if miRNA-221-3p is transferred from primary mouse hepatocytes, which were transfected with miR-221-3p mimic, to primary HSCs, we analyzed miR-221-3p expression in both cell types. We observed significantly higher levels of miR-221-3p only in primary hepatocytes but not in primary HSCs (Supplementary Fig. S1C), thus excluding any passive transfer of miRNA from hepatocytes to HSCs in our co-culture setting. Thus, based on our functional miRNA library screening, and subsequent analyses of TIMP1 secretion and 
fibrogenic markers, we identified miR-221-3p as a potential fibrosis regulator and selected it for further study.

\section{MiR-221-3p is upregulated in primary hepatocytes during liver fibrosis}

Our screen indicated a potential fibrogenic role of miR-221-3p mimic in cultured primary hepatocytes in vitro. To understand the relevance of this finding in liver fibrosis, we determined miR-221-3p levels in a mouse model of liver fibrosis, which was induced by injecting $B A L B / c$ mice with $\mathrm{CCl}_{4}$ for eight weeks. At first, we confirmed liver fibrosis by Sirius red staining (Supplementary Fig. S2A) and mRNA expression analyses of profibrogenic markers such as Col1a1, Acta2 and Tgfbr1, which were found to be increased in the fibrotic mouse livers (Supplementary Fig. S2B).

Since our screen suggested a pro-fibrogenic role of miR-221-3p we reasoned that miR-221-3p levels should be de-regulated during liver fibrosis. We therefore measured miR-221-3p levels in livers of mice injected with $\mathrm{CCl}_{4}$. We observed a high miR-221-3p expression in fibrotic liver tissues by in situ hybridization (Fig. 2A). The increased levels of miR-221-3p in fibrotic liver tissues were confirmed by qPCR analysis (Fig. 2B). To further confirm whether miR-221-3p expression is enhanced in hepatocytes, we purified primary hepatocytes from fibrotic livers by liver perfusion followed by density gradient 
centrifugation. We observed significant upregulation of miR-221-3p in hepatocytes (10.69 fold) isolated from the fibrotic mouse liver tissues compared to hepatocytes obtained from normal healthy liver (Fig. 2C). In addition to hepatocytes, we found significant upregulation of miR-221-3p in HSCs as reported previously ${ }^{22}$, and a mild though not significant increase in Kupffer cells, isolated from fibrotic livers.

Since miR-221-3p is upregulated in hepatocytes during liver fibrosis, we sought to investigate the effect of miR-221-3p inhibition in hepatocytes on liver fibrosis. In order to effectively knock down the expression of miR-221-3p in vivo and in vitro, we used Tough Decoys (TuD). TuDs possess a hairpin structure containing two bulged miRNA targeting sites, which contribute to imperfect base-pairing with the miRNA of interest ${ }^{13,23}$. TuDs have previously been reported to inhibit miRNA function efficiently ${ }^{13}$. We first confirmed downregulation of miR-221-3p upon transfection of primary hepatocytes with Tough Decoy miR-221-3p (TuD-miR-221-3p) (Fig. 2D). Importantly, to examine efficacy of TuD-miR-221-3p at the functional level, we analyzed protein level of PUMA, a known target of miR-221-3p ${ }^{15}$, in primary hepatocytes transfected with TuD-miR-221-3p. We observed increased PUMA levels upon TuD-miR-221-3p transfection (Fig. 2E). These results suggest that the 
TuD-miR-221-3p is able to inhibit miR-221-3p expression efficiently at functional level as well.

MiR-221-3p inhibition in hepatocytes leads to faster resolution of liver fibrosis in vivo

Since, our functional miRNA screening resulted in identification of miR-221-3p and its expression was also upregulated in hepatocytes during fibrosis, we hypothesized that inhibition of miR-221-3p in hepatocytes of fibrotic livers would ameliorate liver fibrosis. To test this hypothesis, we aimed to inhibit miR-221-3p in hepatocytes of fibrotic livers by injecting mice with $1 \times 10^{10}$ adeno-associated virus (AAV) serotype 8 encoding TuD-miR-221-3p (AAV TuD) at week 6 and week 8 of $\mathrm{CCl}_{4}$ treatment (Fig. 3A). Mice were sacrificed one week after the second AAV injection. To suppress miR-221-3p specifically in hepatocytes, we expressed TuD-miR-221-3p under the transcriptional control of hepatocyte-specific transthyretin (Ttr) promoter, which resulted in significant downregulation of miR-221-3p in hepatocytes (Fig. 3B). To confirm functional repression of miR-221-3p in vivo, we detected protein levels of PUMA by immunoblotting. PUMA was found to be upregulated in hepatocytes isolated from fibrotic livers of mice injected with AAV TuD, indicating functional repression of miR-221-3p (Fig. 3C). After confirming miR-221-3p inhibition in 
vivo, we determined fibrosis by histology and Sirius Red staining. We observed reduced liver damage and less Sirius Red stained areas in livers of mice injected with AAV TuD compared to respective controls (Fig. 3D). Correlating with the results of Sirius Red staining, reduced cellular infiltration was also observed in liver tissues of mice injected with AAV TuD compared to those injected with AAV control (Fig. 3D). Moreover, the levels of aspartate aminotransferase (AST), alanine aminotransferase (ALT) and bilirubin were significantly downregulated in serum samples of mice injected with AAV TuD compared to the respective controls (Fig. 3E), suggesting reduced liver injury upon miR-221-3p suppression in vivo.

Since the development of fibrosis is regulated by HSCs, we investigated whether the markers of HSC activation decreased upon the miR-221-3p inhibition. The mRNA levels of profibrogenic markers, Col1a1, Acta2, Tgfbr1 (Fig. 3F), and the total collagen content (Fig. 3G) were significantly downregulated in mice injected with AAV TuD. Quiescent HSCs are activated in response to injury by differentiating into $\alpha$-SMA-expressing myofibroblasts ${ }^{24}$. We observed significantly lower a-SMA and desmin positive cells (Fig. $3 \mathrm{H}$ ) in the livers of mice injected with AAV TuD, suggesting reduced fibrosis upon miR-221-3p inhibition. To further confirm that miR-221 inhibition in hepatocytes 
reduces fibrosis, we isolated HSCs from mice injected either with AAV TuD or AAV control, and measured the expression of Col1a1, Lox and Acta2, which are considered as markers of HSC activation. We found a significantly lower expression of Col1a1, Lox and Acta2 (Fig. 3I) indicating reduced fibrosis upon inhibition of miR-221-3p in hepatocytes. Taken together, in vivo inhibition of miR-221-3p in fibrotic liver by AAV TuD ameliorates liver fibrosis.

To rule out adverse effects of miR-221-3p suppression in normal livers, we performed hematoxylin-eosin (HE) and Sirius Red staining and measured ALT, AST, and bilirubin levels in healthy mice injected with AAV control and AAV TuD. HE, Sirius Red staining, mRNA expression of fibrogenic markers, AST, ALT, and bilirubin levels indicated absence of any liver damage upon administration of AAV TuD (Supplementary Fig. S3). These results suggest that AAV TuD neither caused damage nor induced liver fibrosis in healthy livers and hence may be suitable for miR-221-3p inhibition in the clinic.

CYP2E1 is considered as a key metabolic enzyme for $\mathrm{CCl}_{4}$-induced toxicity. Specifically, CYP2E1 deficiency protects mice against $\mathrm{CCl}_{4}$-induced liver injury $^{25}$. Therefore, to rule out the possibility that AAV TuD administration reduced Cyp2e1 levels and hence exhibited lower fibrosis, we measured Cyp2e1 mRNA by q-PCR. Both, AAV TuD and AAV control group of mice 
showed similar levels of Cyp2e1 (Supplementary Fig. S4) indicating that Cyp2e1 levels did not contribute to attenuated fibrosis in AAV TuD injected mice.

Post-transcriptional regulation of Gnai2 by miR-221-3p during liver fibrosis

To investigate the underlying mechanism leading to amelioration of liver fibrosis upon miR-221-3p inhibition, we performed in silico analyses to identify potential miR-221-3p targets that may play a role in liver fibrosis. We used algorithms such as TargetScan (http://www.targetscan.org/), miRDB (http://mirdb.org/miRDB/), and PicTar (http://pictar.mdc-berlin.de/) to narrow down the potential miR-221-3p target gene list (Supplementary Fig. S5A). To cluster the functions of the candidate genes, we applied DAVID (https://david.ncifcrf.gov/) analyses for functional gene annotation (Supplementary Fig. S5B) to uncover mechanism of amelioration of liver fibrosis upon-miR-221-3p. We identified Gnai2 (G protein alpha inhibiting activity polypeptide 2), which has previously been suggested to play key roles in chronic liver diseases ${ }^{26}$, as a promising candidate. The 3'UTR of Gnai2 contains a binding site for miR-221-3p that is conserved in mouse and human (Fig. 4A). To address whether GNAI2 is regulated during liver fibrosis, we 
analyzed GNAI2 protein levels in hepatocytes isolated from mice with healthy and fibrotic livers. The protein levels of GNAI2 were found to be decreased in hepatocytes from fibrotic livers compared to those from healthy livers (Fig. 4B). This indicates that the decrease in GNAI2 protein levels could be due to upregulated levels of miR-221-3p during liver fibrosis. Consistent with these findings, the Gnai2 mRNA expression and the GNAI2 protein levels were de-repressed in hepatocytes of mice with fibrotic livers injected with AAV TuD (Fig. 4C-D).

To determine whether Gnai2 is indeed regulated by miR-221-3p, we transfected primary mouse hepatocytes with miR-221-3p mimic or scramble control and analyzed the protein levels by immunoblotting. GNAI2 protein levels decreased in cells transfected with miR-221-3p mimic compared to cells transfected with scramble controls (Fig. 4E). To determine whether Gnai2 is a bona fide target of miR-221-3p, we cloned the 3'UTR of Gnai2 into a dual luciferase pmirGLO vector and co-transfected this vector with miR-221-3p mimic or scramble control into primary mouse hepatocytes. The dual luciferase reporter assay showed that the luciferase activity significantly decreased in cells co-transfected with miR-221-3p mimic, confirming that Gnai2 is a novel direct target of miR-221-3p (Fig. 4F). In addition to GNAI2, we examined the 
expression of GNAI3, a member of the $\mathrm{Gi}$ group that is frequently downregulated in liver diseases ${ }^{27}$. The protein level of GNAI3 was not regulated in hepatocytes upon inhibition of miR-221-3p in vivo (Supplementary Fig. S5C), thus indicating that only Gnai2 but not Gnai3 is regulated by miR-221-3p at the post-transcriptional level.

MiR-221-3p inhibition in hepatocytes suppresses HSC activation via

\section{GNAI2}

To address whether modulation of Gnai2 in hepatocytes regulates the activation of HSCs, we established transwell co-cultures of primary mouse hepatocytes and primary mouse HSCs. Hepatocytes were cultured in transwells overnight and transfected with a GNAI2 expression vector (pCMV-GNAI2-GFP). After 12 hours of transfection, the transwells were placed on top of primary mouse HSCs. The upregulated expression of Gnai2 mRNA in primary mouse hepatocytes was confirmed at 24 hours post transfection (Supplementary Fig. S6A). The P75 neurotrophin receptor (P75NTR) is expressed at low levels in quiescent HSCs but is rapidly increased after its activation ${ }^{28,29}$. We then examined P75ntr, Acta2, Des mRNA expression levels by qPCR and protein levels by immunofluorescence staining. Both, mRNA and protein levels of P75ntr, Acta2 and Des were significantly 
downregulated in primary mouse HSCs co-cultured with GNAI2-overexpressing primary mouse hepatocytes (Fig. 5A-B).

To further confirm whether GNAI2 contributes to the anti-fibrotic effect observed upon miR-221-3p inhibition, we co-cultured primary mouse HSCs with primary mouse hepatocytes, which were co-transfected with miR-221-3p inhibitor and siRNA against Gnai2. We found that anti-fibrotic effects observed upon miR-221-3p inhibitor were moderately, but significantly diminished when hepatocytes were co-transfected with Gnai2 siRNA (Fig. 5C). These results suggest that GNAI2 indeed contributes to reduced HSC activation upon miR-221-3p inhibition in hepatocytes.

To address, whether Gnai2 overexpression affects functionality of hepatocytes, we analyzed expression of functional markers of hepatocytes such as $A l b$, Krt18 and Hnf4a. We found similar levels of all three hepatocyte markers upon Gnai2 overexpression compared to control (Supplementary Fig. S6B), indicating overexpression of Gnai2 does not affect hepatocyte functions.

We next sought to address the regulatory mechanism of HSC activation by GNAI2. Chemokine CCL2, one of the key regulators of inflammation and liver fibrosis $^{30}$, has previously been reported to be elevated upon knockdown of Gnai2 in mice ${ }^{31}$. Since we observed regulation of Gnai2 by miR-221-3p, we 
therefore reasoned that CCL2 could be a key component of miR-221-3p-GNAI2 signaling. We found a downregulation of Cc/2 mRNA expression in hepatocytes, but not in non-parenchymal cells from mice with liver fibrosis injected with AAV TuD (Fig. 6A-B and Supplementary Fig. S7). Consistent with these results, overexpression of GNAI2 in primary mouse hepatocytes led to a significant reduction in CCL2 secretion (Fig. 6C).

To confirm whether reduction in CCL2 is exerted by miR-221-3p inhibition, we collected supernatant from the culture medium of transfected primary mouse hepatocytes with miR-221-3p inhibitors. The ELISA results indeed confirmed reduction in secreted CCL2 levels (Fig. 6D). In accordance with these results, siRNA-based knockdown of Gnai2 mRNA (Fig. 6E) led to a significant increase in secreted CCL2 levels (Fig. 6F).

To further verify the direct effects of CCL2 on HSCs, we treated primary mouse HSCs with $100 \mathrm{ng} / \mathrm{ml}$ CCL2 for 48 hours and measured expression of fibrotic markers by qPCR and immunofluorescence staining. The expression of Col1a1, Acta2 and Tgfbr1 and protein levels of p75NTR, aSMA, and Desmin were significantly upregulated in primary mouse HSCs treated with CCL2 (Fig. 6G-H). Consistent with these results, primary mouse HSCs treated with the supernatant collected from primary mouse hepatocytes transfected with 
miR-221-3p inhibitors showed significant downregulation of Col1a1, Acta2 and Tgfbr1 by qPCR and protein levels of p75NTR, aSMA, and Desmin (Fig. 6I-J). Taken together, our data suggest that upregulation of GNAI2 inhibited the expression of both Cc/2 mRNA and CCL2 protein levels in hepatocytes that led to the inhibition of HSC activation and amelioration of liver fibrosis.

In addition to being a potent activator for HSC, CCL2 promotes chemotaxis for monocytes derived from bone marrow. We therefore, investigated whether miR-221-3p mitigated fibrosis not only by reducing HSC activation but also by reducing monocyte recruitment. To address this, we analyzed monocyte recruitment by FACS (Supplementary Fig. S8A) as well as IHC (Supplementary Fig. S8B) for CD11b and F4/80. We observed lower number of $\mathrm{CD} 11 \mathrm{~b}$ and F4/80 cells indicating reduced monocyte recruitment indeed contributed to mitigation of liver fibrosis upon miR-221-3p inhibition in hepatocytes.

We next asked whether attenuation of fibrosis upon miR-221-3p inhibition is restricted to $\mathrm{CCl}_{4}$ model only. To answer this, we injected AAV TuD in mice fed with 3,5-di diethoxycarbonyl-1,4-dihydrocollidine (DDC)-containing diet, a model of cholestasis-induced fibrosis (Fig. 7A). We analyzed fibrosis by histology, Sirius Red staining, hydroxyproline assay, fibrogenic markers' 
mRNA analyses in whole liver and isolated HSCs and immunofluorescence staining for aSMA, p75NTR and Desmin (Fig. 7B-F and Supplementary Fig. S9A). The absence of dramatic changes in the expression of fibrogenic genes in isolated HSCs (Fig. 7F) might be due to cellular contamination resulting from the technically challenging perfusion of livers from DDC-fed mice (Supplementary Fig. S9B). Taken together, as with the $\mathrm{CCl}_{4}$ model, we observed attenuation of DDC-induced liver fibrosis upon miR-221-3p inhibition. To examine whether our findings are applicable to human hepatocytes, we first inhibited miR-221-3p in primary human hepatocytes by transfecting them with miR-221-3p inhibitor, and collected medium for the next 48 hours (Figure 8A). We then treated primary human myofibroblasts with culture medium collected from primary human hepatocytes (Fig. 8A). Subsequent q-PCR analyses for Col1a1, Acta2 and Tgfbr1 (Fig. 8B) and immunofluorescence staining for p75NTR, aSMA, Desmin and LOX (Fig. 8C) showed significantly reduced expression of all markers in primary human myofibroblasts. These results indicate that culture medium collected from miR-221-3p inhibited-primary human hepatocytes is able to reduce activation of primary human myofibroblast.

Finally, we investigated if miR-221-3p expression is changed in primary human 
hepatocytes isolated from fibrotic livers. In concert with mouse data, miR-221-3p expression by q-PCR analyses showed an upregulation in primary human hepatocytes isolated from fibrotic livers compared to non-fibrotic control livers (Fig. 8D). Furthermore, miR-221-3p upregulation correlated with reduced GNAI2 and concomitant upregulation of CCL2 protein levels in primary human hepatocytes obtained from fibrotic livers compared to respective controls (Fig. 8E).

\section{Discussion}

Current anti-fibrotic therapies mainly focus on HSCs such as the prevention of HSC activation, inhibition of fibrogenesis, and the resolution of fibrosis ${ }^{32}$. However, hepatocytes consist of up to $80 \%$ of the liver mass and are responsible for mediating a broad range of interactions between different cell types such as HSCs, Kupffer cells, and epithelial cells ${ }^{20}$. Our study highlights the therapeutic potential of targeting hepatocytes for treating liver fibrosis. We demonstrate that miR-221-3p is upregulated in hepatocytes during liver fibrosis. Importantly, in vivo knockdown of miR-221-3p by AAV TuD specifically in hepatocytes not only suppresses $\mathrm{HSC}$ activation but also alleviates $\mathrm{CCl}_{4}$ and DDC-induced liver fibrosis in mice. Unlike other methods of RNA silencing 
that lead to systemic effects, hepatotrophic AAV TuD specifically targets hepatocytes and spares the off-target effects ${ }^{15,33,34}$. Therefore, as the most abundant cell type in the liver, the hepatocytes, not only play an important role in regulating the activation of HSCs in liver damage, but also have an advantage of being a therapeutic target of liver fibrosis.

Mechanistically, we identified Gnai2 as a novel miR-221-3p target. GNAI2, which belongs to the inhibitory $G$ alpha family, is involved in signaling pathways related to cell cycle and hormone regulation ${ }^{35,36}$. GNAI2 is downregulated in HCC and knock-down of GNAI2 expression significantly increases cell invasion and migration in vitro suggesting GNAI2 plays a role in suppressing metastasis ${ }^{26}$. Consistent with upregulation of miR-221-3p expression in mouse model of liver fibrosis, GNAI2 levels are reduced in liver fibrosis indicating its pathophysiological relevance. Importantly, GNAI2-overexpressing hepatocytes suppressed the HSC activation in co-culture experiments in vitro.

The CCR2-CCL2 signaling axis is a major regulator of HSC activation ${ }^{20}$. It has been shown that either blocking of CCL2 in rats or depletion of CCR2 in mice genetically $^{37}$ as well as pharmacological inhibition of CCL2 remarkably attenuates liver fibrosis ${ }^{38}$. Further, the high expression of CCL2 in HCC is 
associated with the poor prognosis of patients ${ }^{30}$. Furthermore, the loss of GNAI2 in Gnai2 $^{-/-}$mice leads to increased CCL2 in chronic inflammation ${ }^{31}$. Importantly, we found that overexpression of GNAI2 in primary mouse hepatocytes results in reduced levels of CCL2. Thus, our findings indicate that miR-221-3p inhibition in hepatocytes suppressed the activation of HSCs via regulation of the miR-221-GNAI2-CCl2 axis. Our data revealed that decreased $\mathrm{CCl}$ levels upon miR-221-3p inhibition contribute to amelioration of fibrosis not only by reducing HSC activation but also lowering the monocyte recruitment in liver. We thus, we demonstrate for the first time that knocking down the expression of miR-221-3p in hepatocytes leads to decreased activation of HSCs and, finally amelioration of liver fibrosis. In concert with data obtained from mouse experiments, we demonstrated that miR-221-3p inhibition in primary human hepatocytes was capable of reducing fibrogenic markers in primary human myofibroblasts indicating translational relevance of our findings. Although our in vitro experiments suggest a direct interaction between hepatocytes and myofibroblasts, there remains a possibility that protective activity of AAV TUD in hepatocytes also suppresses liver injury. In fact, the lower levels of ALT, AST and bilirubin in mice injected with AAV TUD indicate less hepatocyte injury, which in turn may result in a 
reduced fibrosis response. It is important to mention that sustained overexpression of miR-221 for several months in mice promotes tumorigenicity in liver ${ }^{39}$. Furthermore, silencing of miR-221 by synthetic anti-miR is able to hinder hepatocellular carcinoma progression $^{40}$, indicating reduced hepatocellular injury. Therefore, in light of our results and previously published data ${ }^{39,40}$, we cannot exclude the possibility that reduced injury of hepatocytes may have also contributed to the observed mitigation of fibrosis.

Taken together, we show that suppression of miR-221-3p in hepatocytes of fibrotic livers leads to faster resolution of the deposited extracellular matrix. Furthermore, downregulation of miR-221-3p induces the expression of GNAI2 in hepatocytes, which in turn inhibits CCL2 secretion, and hence leads to reduced HSC activation and amelioration of liver fibrosis. Our study highlights the promising potential of miRNA-221-3p as a therapeutic intervention for liver disease.

\section{Acknowledgements}

The study was supported by the Deutsche Forschungsgemeinschaft (DFG SH640/1-2, DFG EXC 62/2 and SFB-738), Gilead Sciences International Research Scholars Program in Liver Diseases, PLUS3 programme of 
Boehringer Ingelgeim and the Bundesministerium für Bildung und Forschung (Biodisc 6, START-MSC II). A.B. is supported from Deutsche Krebshilfe grant (111147).

\section{References}

1 Lozano, R. et al. Global and regional mortality from 235 causes of death for 20 age groups in 1990 and 2010: a systematic analysis for the Global Burden of Disease Study 2010. Lancet 380, 2095-2128, doi:10.1016/S0140-6736(12)61728-0 (2012).

2 Tsochatzis, E. A., Bosch, J. \& Burroughs, A. K. Liver cirrhosis. Lancet 383, 1749-1761, doi:10.1016/S0140-6736(14)60121-5 (2014).

3 Blachier, M., Leleu, H., Peck-Radosavljevic, M., Valla, D. C. \& Roudot-Thoraval, F. The burden of liver disease in Europe: a review of available epidemiological data. J Hepatol 58, 593-608, doi:S0168-8278(12)00924-5 [pii]

10.1016/j.jhep.2012.12.005 (2013).

4 Koyama, Y. \& Brenner, D. A. Liver inflammation and fibrosis. J Clin Invest 127, 55-64, doi:10.1172/JCI88881 (2017).

5 Schuppan, D. \& Kim, Y. O. Evolving therapies for liver fibrosis. The 
Journal of clinical investigation 123, 1887-1901, doi:10.1172/JCI66028 (2013).

6 Lee, U. E. \& Friedman, S. L. Mechanisms of hepatic fibrogenesis. Best practice \& research. Clinical gastroenterology 25, 195-206, doi:10.1016/j.bpg.2011.02.005 (2011).

7 Szabo, G. \& Bala, S. MicroRNAs in liver disease. Nature reviews. Gastroenterology \& hepatology 10, 542-552, doi:10.1038/nrgastro.2013.87 (2013).

8 Ambros, V. The functions of animal microRNAs. Nature 431, 350-355, doi:10.1038/nature02871 (2004).

9 Lakner, A. M. et al. Inhibitory effects of microRNA 19b in hepatic stellate cell-mediated fibrogenesis. Hepatology 56, 300-310, doi:10.1002/hep.25613 (2012).

10 Ludtke, T. H. et al. Tbx2 controls lung growth by direct repression of the cell cycle inhibitor genes Cdkn1a and Cdkn1b. PLoS genetics $\mathbf{9}$ e1003189, doi:10.1371/journal.pgen.1003189 (2013).

11 Wilkinson, D. G. \& Nieto, M. A. Detection of messenger RNA by in situ hybridization to tissue sections and whole mounts. Methods in enzymology 225, 361-373 (1993). 
12 Moorman, A. F., Houweling, A. C., de Boer, P. A. \& Christoffels, V. M. Sensitive nonradioactive detection of mRNA in tissue sections: novel application of the whole-mount in situ hybridization protocol. The journal of histochemistry and cytochemistry : official journal of the Histochemistry Society 49, 1-8, doi:10.1177/002215540104900101 (2001).

13 Bak, R. O., Hollensen, A. K., Primo, M. N., Sorensen, C. D. \& Mikkelsen, J. G. Potent microRNA suppression by RNA Pol II-transcribed 'Tough Decoy' inhibitors. RNA 19, 280-293, doi:10.1261/rna.034850.112 (2013).

14 Gellhaus, K., Cornu, T. I., Heilbronn, R. \& Cathomen, T. Fate of recombinant adeno-associated viral vector genomes during DNA double-strand break-induced gene targeting in human cells. Human gene therapy 21, 543-553, doi:10.1089/hum.2009.167 (2010).

15 Sharma, A. D. et al. MicroRNA-221 regulates FAS-induced fulminant liver failure. Hepatology 53, 1651-1661, doi:10.1002/hep.24243 (2011).

16 Maschmeyer, P., Flach, M. \& Winau, F. Seven steps to stellate cells. J Vis Exp, doi:10.3791/2710 (2011).

17 Kleine, M. et al. Explanted diseased livers - a possible source of 
metabolic competent primary human hepatocytes. PLoS One $\mathbf{9}$, e101386, doi:10.1371/journal.pone.0101386 (2014).

18 Borst, K. et al. Type I interferon receptor signaling delays Kupffer cell replenishment during acute fulminant viral hepatitis. J Hepatol, doi:10.1016/j.jhep.2017.11.029 (2017).

19 Meurer, S. K. et al. Overexpression of endoglin modulates TGF-beta1-signalling pathways in a novel immortalized mouse hepatic stellate cell line. PLoS One 8, e56116, doi:10.1371/journal.pone.0056116

PONE-D-12-22919 [pii] (2013).

20 Pellicoro, A., Ramachandran, P., Iredale, J. P. \& Fallowfield, J. A. Liver fibrosis and repair: immune regulation of wound healing in a solid organ. Nature reviews. Immunology 14, 181-194, doi:10.1038/nri3623 (2014).

21 Iredale, J. P. et al. Tissue inhibitor of metalloproteinase-1 messenger RNA expression is enhanced relative to interstitial collagenase messenger RNA in experimental liver injury and fibrosis. Hepatology 24, 176-184, doi:10.1002/hep.510240129 (1996).

22 Ogawa, T. et al. MicroRNA-221/222 upregulation indicates the activation of stellate cells and the progression of liver fibrosis. Gut $\mathbf{6 1}$, 
1600-1609, doi:10.1136/gutjnl-2011-300717 (2012).

23 Haraguchi, T., Ozaki, Y. \& Iba, H. Vectors expressing efficient RNA decoys achieve the long-term suppression of specific microRNA activity in mammalian cells. Nucleic acids research 37 , e43, doi:10.1093/nar/gkp040 (2009).

24 Iwaisako, K. et al. Origin of myofibroblasts in the fibrotic liver in mice. Proceedings of the National Academy of Sciences of the United States of America 111, E3297-3305, doi:10.1073/pnas.1400062111 (2014).

25 Wong, F. W., Chan, W. Y. \& Lee, S. S. Resistance to carbon tetrachloride-induced hepatotoxicity in mice which lack CYP2E1 expression. Toxicol Appl Pharmacol 153, 109-118, doi:10.1006/taap.1998.8547 (1998).

26 Yao, J. et al. MicroRNA-30d promotes tumor invasion and metastasis by targeting Galphai2 in hepatocellular carcinoma. Hepatology 51 , 846-856, doi:10.1002/hep.23443 (2010).

27 Zhang, Y. et al. GNAI3 inhibits tumor cell migration and invasion and is post-transcriptionally regulated by miR-222 in hepatocellular carcinoma. Cancer letters 356, 978-984, doi:10.1016/j.canlet.2014.11.013 (2015).

28 Reetz, J. et al. Development of Adenoviral Delivery Systems to Target 
Hepatic Stellate Cells In Vivo. PLoS One 8, e67091,

doi:10.1371/journal.pone.0067091

PONE-D-12-32872 [pii] (2013).

29 Asai, K. et al. Activated hepatic stellate cells overexpress p75NTR after partial hepatectomy and undergo apoptosis on nerve growth factor stimulation. Liver international : official journal of the International Association for the Study of the Liver 26, 595-603, doi:10.1111/j.1478-3231.2006.01267.x (2006).

$30 \mathrm{Li}, \mathrm{X}$. et al. Targeting of tumour-infiltrating macrophages via CCL2/CCR2 signalling as a therapeutic strategy against hepatocellular carcinoma. Gut, doi:10.1136/gutjnl-2015-310514 (2015).

31 Gotlind, Y. Y. et al. Interplay between T(h)1 and T(h)17 effector T-cell pathways in the pathogenesis of spontaneous colitis and colon cancer in the Galphai2-deficient mouse. International immunology 25, 35-44, doi:10.1093/intimm/dxs089 (2013).

32 Lee, Y. A., Wallace, M. C. \& Friedman, S. L. Pathobiology of liver fibrosis: a translational success story. Gut 64, 830-841, doi:10.1136/gutjnl-2014-306842 (2015).

33 Di Martino, M. T. et al. In vitro and in vivo activity of a novel locked 
nucleic acid (LNA)-inhibitor-miR-221 against multiple myeloma cells.

PloS one 9, e89659, doi:10.1371/journal.pone.0089659 (2014).

34 Yuan, Q. et al. MicroRNA-221 overexpression accelerates hepatocyte proliferation during liver regeneration. Hepatology 57, 299-310, doi:10.1002/hep.25984 (2013).

35 Neves, S. R., Ram, P. T. \& lyengar, R. G protein pathways. Science 296, 1636-1639, doi:10.1126/science.1071550 (2002).

36 Raymond, J. R., Jr., Appleton, K. M., Pierce, J. Y. \& Peterson, Y. K. Suppression of GNAI2 message in ovarian cancer. Journal of ovarian research 7, 6, doi:10.1186/1757-2215-7-6 (2014).

37 Mitchell, C. et al. Dual role of CCR2 in the constitution and the resolution of liver fibrosis in mice. The American journal of pathology 174, 1766-1775, doi:10.2353/ajpath.2009.080632 (2009).

38 Baeck, C. et al. Pharmacological inhibition of the chemokine CCL2 (MCP-1) diminishes liver macrophage infiltration and steatohepatitis in chronic hepatic injury. Gut 61, 416-426, doi:10.1136/gutjnl-2011-300304 (2012).

39 Callegari, E. et al. Liver tumorigenicity promoted by microRNA-221 in a mouse transgenic model. Hepatology 56, 1025-1033, 
doi:10.1002/hep.25747 (2012).

40 Park, J. K. et al. miR-221 silencing blocks hepatocellular carcinoma and promotes survival. Cancer research 71, 7608-7616, doi:10.1158/0008-5472.CAN-11-1144 (2011).

\section{Figure legends:}

Fig. 1. The miRNA mimic library screening identifies miR-221-3p as potential fibrosis regulator. (A) A scheme shows the experimental design of miRNA mimic library screening and subsequent steps to identify miRNAs, whose modulation in primary hepatocytes is capable of regulating fibrosis. (B) The fold change in GFP intensity upon transfection of 302 miRNA mimics is depicted as readout of fibrosis. The GFP intensity of scramble control (scr) is set as $1(n=3)$. (C) Fifteen miRNAs transfected in primary mouse hepatocytes contributed to significantly increased GFP/WST1 intensity of Col-GFP HSCs than respective scramble control. The GFP/WST1 intensity of scramble is set as $1(n=4)$. (D) ELISA results show that miR-221-3p mimic transfection led to higher TIMP1 concentration than that of scramble. (E) A scheme shows the experimental design of confirmatory experiments performed for validation of miR-221-3p. Primary hepatocytes were transfected with miR-221-3p mimic 
and subsequently, co-cultured with primary mouse HSCs. TIMP1 ELISA and q-PCR analyses revealed significantly upregulated TIMP1 levels and fibrosis markers, respectively. Error bars represent \pm SEM. One-way ANOVA was used for statistical evaluation. $\left({ }^{*} p<0.05 ;{ }^{* *} p<0.01\right)$.

Fig. 2 MiR-221-3p is upregulated in liver fibrosis. (A) In situ hybridization indicates higher miR-221-3p expression in fibrotic mouse liver tissues compared to miR-221-3p expression in control (healthy mouse livers). Q-PCR confirms significantly increased miR-221-3p expression in (B) liver and (C) hepatocytes and HSCs, but not Kupffer cells of a $\mathrm{CCl}_{4}$-induced mouse liver fibrosis model compared to non-fibrotic livers ( $n=3$ mice/group). (D) Significant downregulation of miR-221-3p by TuD-miR-221-3p in primary hepatocytes led to $(E)$ upregulation of PUMA, a known miR-221-3p target gene in vitro $(n=3)$. Scale bars: $50 \mu \mathrm{m}$. Error bars represent \pm SEM. Two-tailed Student's t-test was used for statistical evaluation. $\quad\left({ }^{*} p<0.05 ;{ }^{* *} p<0.01 ;{ }^{* *} p<0.001\right)$.

Fig. 3. Inhibition of miR-221-3p in hepatocytes ameliorates liver fibrosis in vivo. (A) A scheme of liver fibrosis induction and time points of $A A V$ injections. (B) Q-PCR result shows that miR-221-3p is significantly 
downregulated in hepatocytes upon AAV TuD transfection ( $n=5$ mice/group). (C) Downregulation of miR-221-3p through AAV TuD leads to increase in PUMA as shown by immunoblotting $(n=3)$. The proteins were prepared from isolated hepatocytes. Two right lanes were loaded with protein lysates obtained from fibrotic livers. (D) AAV TuD suppresses liver fibrosis in vivo as observed by fewer micro nodules (gross morphology), less cellular infiltration (HE staining), and significantly reduced area of liver fibrosis (Sirius Red staining) ( $n=25$ fields/group). (E) Significantly lower levels of AST, ALT, and bilirubin in serum from mice injected with AAV TuD indicates reduced liver injury ( $\mathrm{n}=4 \mathrm{mice/group).} \mathrm{Magnification:} 100 X$. Error bars represent $\pm \operatorname{SEM}\left({ }^{*} \mathrm{p}<\right.$ 0.05). (F) Q-PCR shows that the expression of fibrotic markers Col1a1, Acta2 and Tgfbr1 was significantly downregulated ( $n=4$ mice/group). (G) The amount of collagen decreased in mice injected with AAV TuD. $(H)$ The protein levels of aSMA and Desmin are significantly downregulated in fibrotic livers from mice injected with AAV TuD. The co-staining of aSMA and Desmin further indicated reduced fibrosis in mice injected with AAV TuD. (I) The q-PCR analyses show significantly lower levels of Col1a1, Lox and Acta2 in primary mouse HSCs isolated from livers of mice injected with AAV-TuD. CTCF: Corrected Total Cell Fluorescence. Magnification: 200X (aSMA and Desmin), ( $n=25$ fields/group). 
Error bars represent \pm SEM. One-way ANOVA was used for statistical evaluation. $\left({ }^{*} p<0.05 ;{ }^{* *} p<0.01 ;{ }^{* *} p<0.001\right)$

Fig. 4. Gnai2 is a novel target gene of miR-221-3p. (A) A targeted sequence of 3'UTR of Gnai2 by miR-221-3p is shown. (B) GNAI2 is significantly downregulated in primary hepatocytes isolated from fibrotic liver tissues in vivo. AAV TuD injection leads to upregulation of Gnai2 (C) mRNA and (D) protein in isolated primary hepatocytes ( $n=7$ mice/group). (E) Transfection of miR-221-3p mimic in primary hepatocytes leads to a decrease in GNAI2 protein levels compared to respective scramble control $(n=5)$. (F) Dual luciferase reporter assay shows that miR-221-3p directly binds to 3'UTR of Gnai2, suggesting Gnai2 is a direct target of miR-221-3p. Data are shown as fold change and compared with the control group set as one. RLU: relative luminescence units $(n=4)$. Error bars represent \pm SEM. One-way ANOVA was used for statistical evaluation. $\left({ }^{*} p<0.05\right)$.

Fig. 5. MiR-221-3p inhibition in hepatocytes reduces HSC activation via GNAI2. Downregulation of p75ntr, Acta2 and Des (A) mRNA (n=3) and (B) protein levels in primary mouse HSCs co-cultured with GNAI2 overexpressing 
primary mouse hepatocytes in vitro. (C) Immunofluorescence staining show de-repression of HSC activation markers when primary mouse HSCs were co-cultured with primary mouse hepatocytes, which were co-transfected with miR-221-3p inhibitor and Gnai2 siRNA. CTCF: corrected total cell fluorescence ( $n \geq 4$ fields/ group). Magnification: 100X. Error bars represent \pm SEM. One-way ANOVA was used for statistical evaluation. $\left({ }^{*} p<0.05 ;{ }^{* *} p<0.01\right.$; $\left.{ }^{* * *} p<0.001\right)$

Fig. 6. CCL2 regulates the activation of HSCs. The mRNA expression of Ccl2 is decreased in (A) primary mouse hepatocytes but not in (B) non-parenchymal cells in vivo ( $n=7$ mice/group). (C) Overexpression of GNAI2 or (D) downregulation of miR-221-3p lowered the CCL2 secretion in primary mouse hepatocytes in vitro $(n=9)$. (E) Knockdown of GNAI2 leads to $(F)$ increased CCL2 secretion from hepatocytes ( $n=3)$. (G) CCL2 increased levels of fibrotic markers, Col1a1, Acta2 and Tgfbr1 $(\mathrm{n}=4)$ and $(\mathrm{H})$ protein expression of HSC activation markers, p75NTR, aSMA, Desmin and LOX ( $n=21$ fields/group). (I) Reduced secretion of CCL2 upon treatment with miR-221-3p inhibitors facilitates suppression of of fibrotic markers, Col1a1, Acta2 and Tgfbr1 $(n=3)$ and $(\mathrm{J})$ protein expression of HSC activation markers, p75NTR, 
aSMA, Desmin and LOX ( $\mathrm{n}=15$ fields/group). CTCF: corrected total cell fluorescence. Magnification: 100X. Error bars represent \pm SEM. One-way ANOVA was used for statistical evaluation. $\left({ }^{*} p<0.05 ;{ }^{* *} p<0.001 ;{ }^{* *} p<0.01\right)$.

Fig. 7: Inhibition of miR-221-3p in hepatocytes mitigates DDC-induced liver fibrosis in vivo. (A) A scheme of DDC-induced liver fibrosis and time points of AAV injections. (B) Q-PCR result shows significantly downregulation of miR-221-3p hepatocytes upon AAV TuD transfection (Healthy $n=3 /$ group, AAV Control $n=5$ mice/group, AAV TuD $n=4$ mice/group). (C,D) Suppression of liver fibrosis in vivo by AAV TuD as observed by less micro nodules in gross morphology (C), less cellular infiltration (HE staining), and significantly reduced area of liver fibrosis shown by Sirius Red staining (D) ( $n=21$ fields/group). (E) The amount of collagen significantly decreased in mice injected with AAV TuD. (F) Q-PCR shows that the expression of fibrotic markers Col1a1, Acta2 and Lox was significantly downregulated in both livers and primary moues HSCs (healthy $n=3$ mice/group, AAV Control $n=5$ mice/group, AAV TuD $n=4$ mice/group). (G) Significant downregulation of aSMA and Desmin in fibrotic livers from mice injected with AAV TuD. The co-staining of aSMA and Desmin further revealed lower fibrosis in mice injected with AAV TuD. CTCF: 
Corrected Total Cell Fluorescence. Magnification: 200X (aSMA and Desmin), ( $n=9$ fields/group). Error bars represent \pm SEM. One-way ANOVA was used for statistical evaluation. $\left({ }^{*} p<0.05 ;{ }^{* *} p<0.01 ;{ }^{* * *} p<0.001\right)$

Fig. 8: Altered expression of miR-221-3p in primary human hepatocytes suppresses fibrogenic markers in primary human myofibroblasts. (A) Schematic of experimental design showing primary human hepatocytes were transfected with miR-221-3p inhibitor. The primary human myofibroblasts were then exposed to the medium collected from miR-221-3p inhibitor transfected-primary human hepatocytes. (B) The q-PCR analyses $(n=3)$ and (C) immunofluorescence staining for fibrogenic markers show reduced expression ( $n \geq 6$ fields/group). (D) Measurement of miR-221-3p expression by q-PCR in primary human hepatocytes isolated from human fibrotic patients $(n=3)$ and controls (non fibrotic livers, $n=3)$. (E) Immunofluorescence staining for GNAI2 and CCL2 on primary human hepatocytes collected on slides after cytospin. CTCF: corrected total cell fluorescence. Magnification: 100X. Error bars represent \pm SEM. One-way ANOVA was used for statistical evaluation. $\left({ }^{*} p<0.05 ;{ }^{* *} p<0.01 ;{ }^{* * *} p<0.001\right)$. 


\section{AFigure 1}

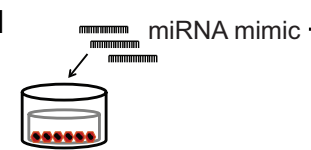

primary mouse hepatocytes in a 96-well insert

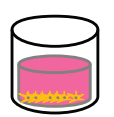

Col-GFP HSCs in a 96-well receiving well)

$\mathrm{B}$

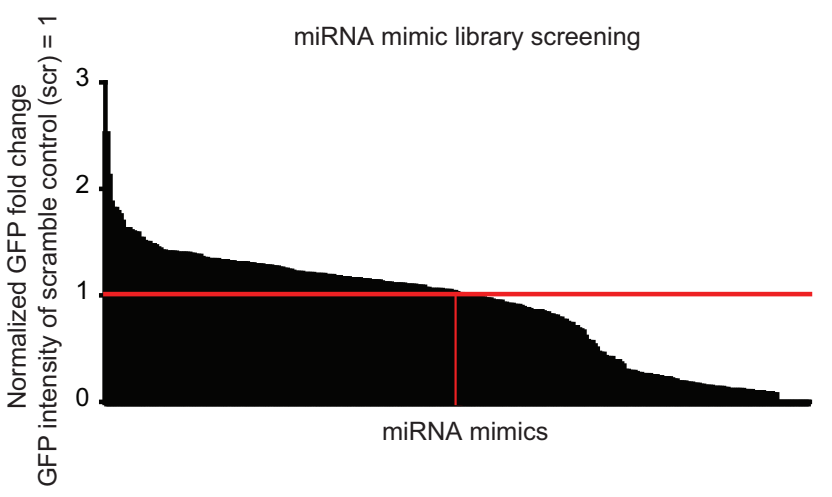

C

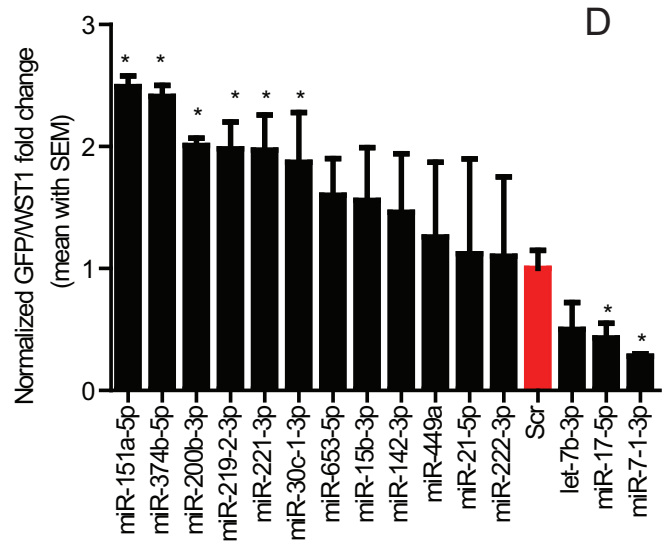

$\stackrel{\text { GFP measurement }}{\longrightarrow} 15$ miRNAs $\stackrel{\text { TIMP1 }}{\longrightarrow}$ miR-221-3p

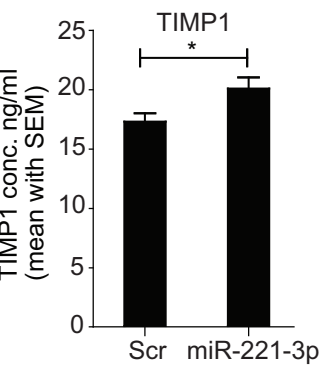

E

Primary mouse HSCs co-cultured with miR-221-3p mimic-transfected primary mouse hepatocytes
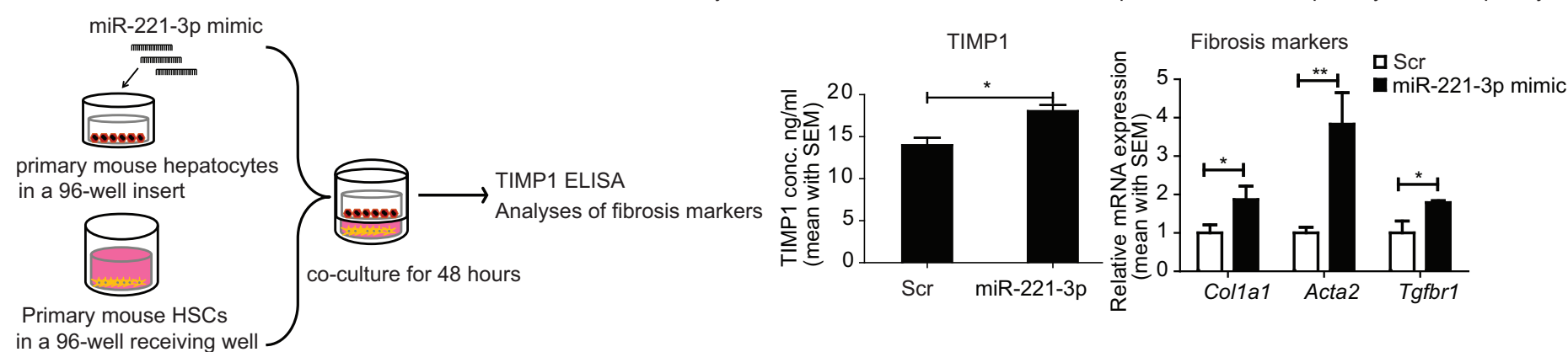

Figure1 


\section{Figure 2}

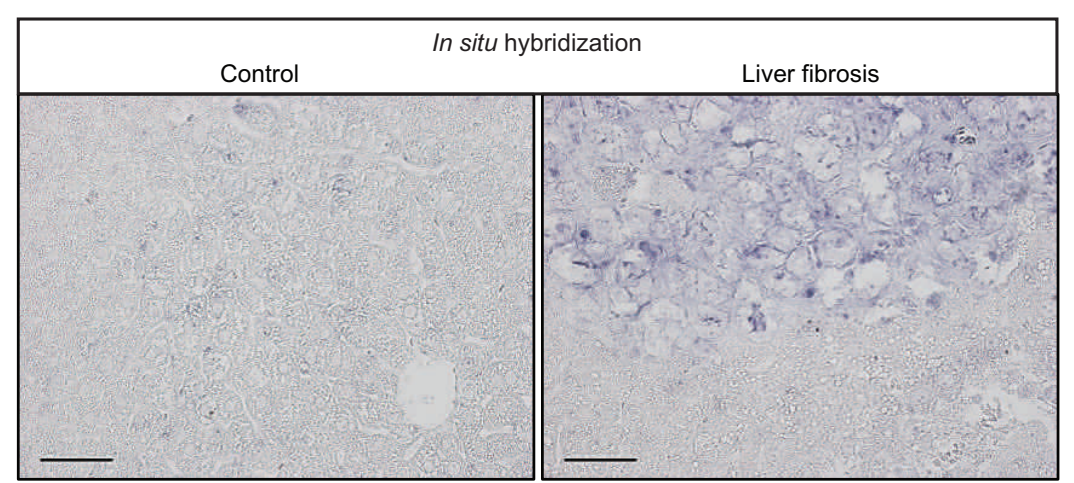

C

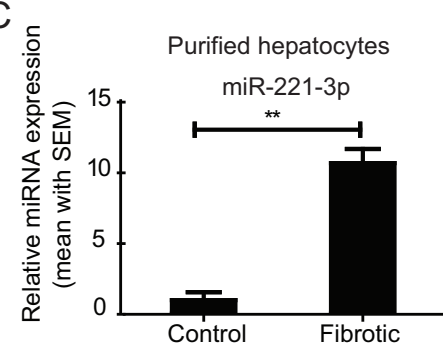

D

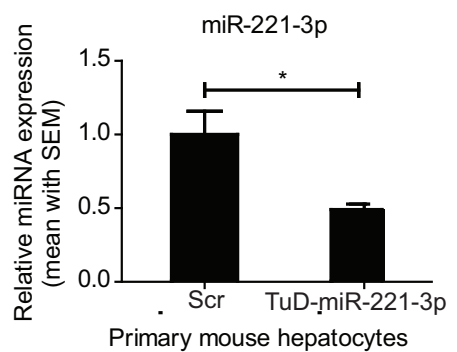

E
B

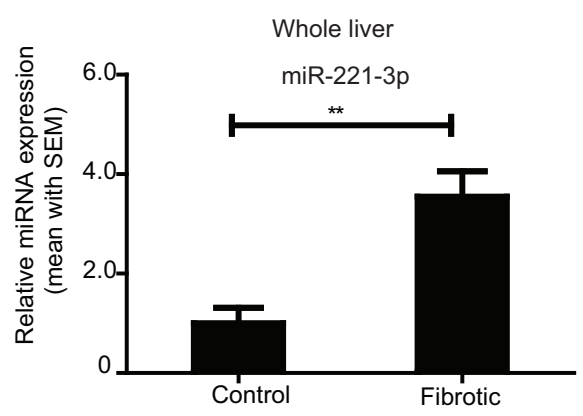

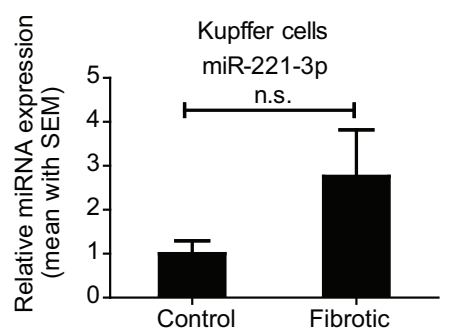

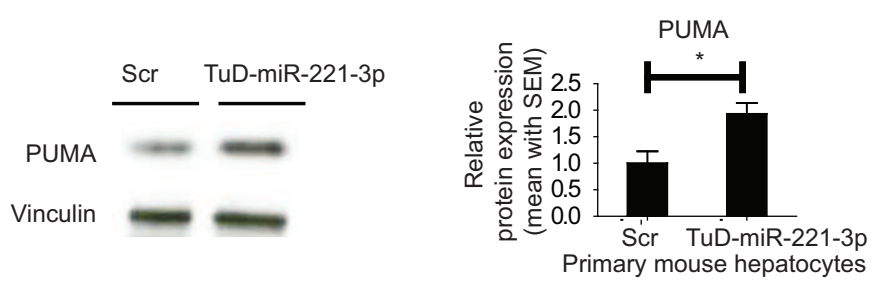




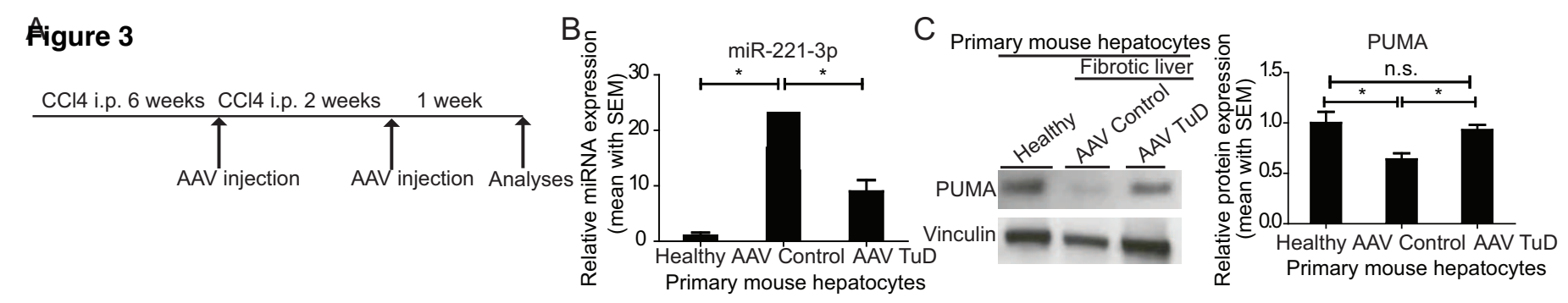

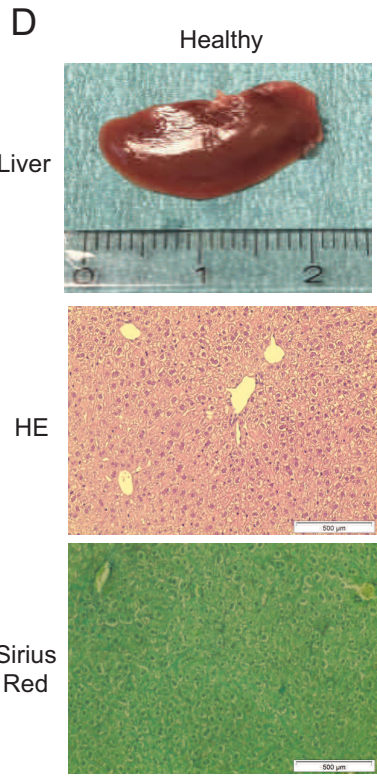

F

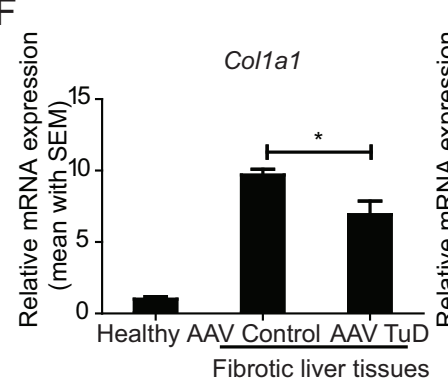

AAV Control
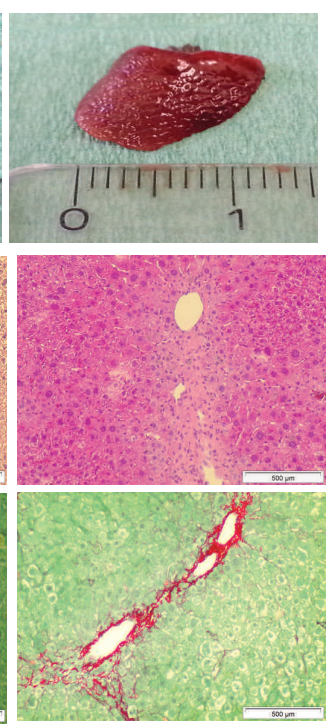

AAV TuD

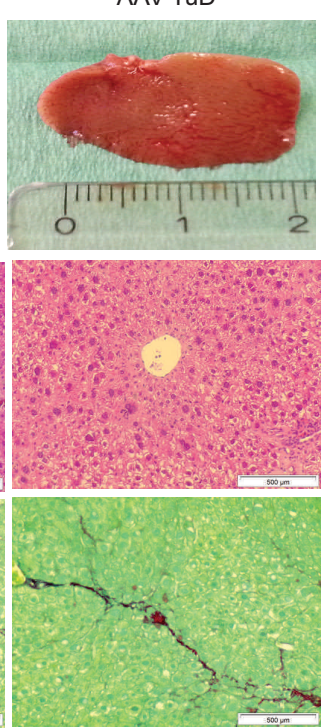

E
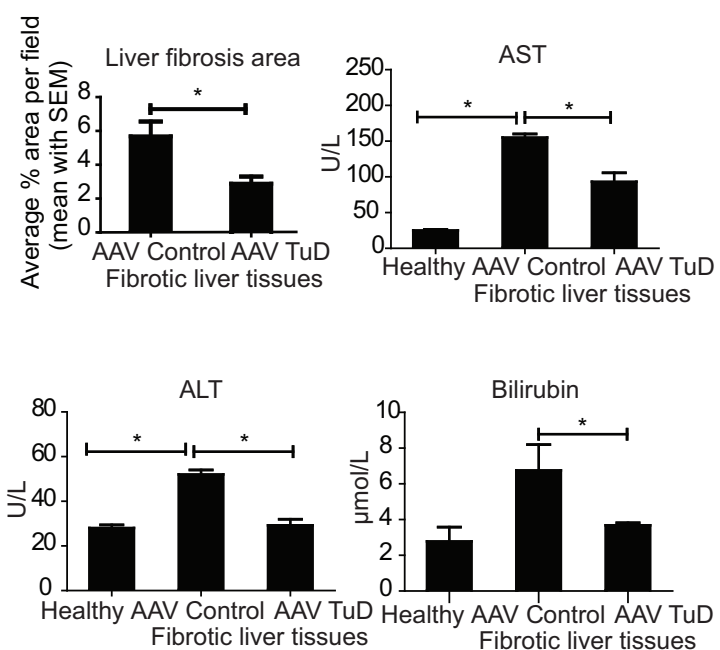

G

Tgfbr1

Hydroxyproline
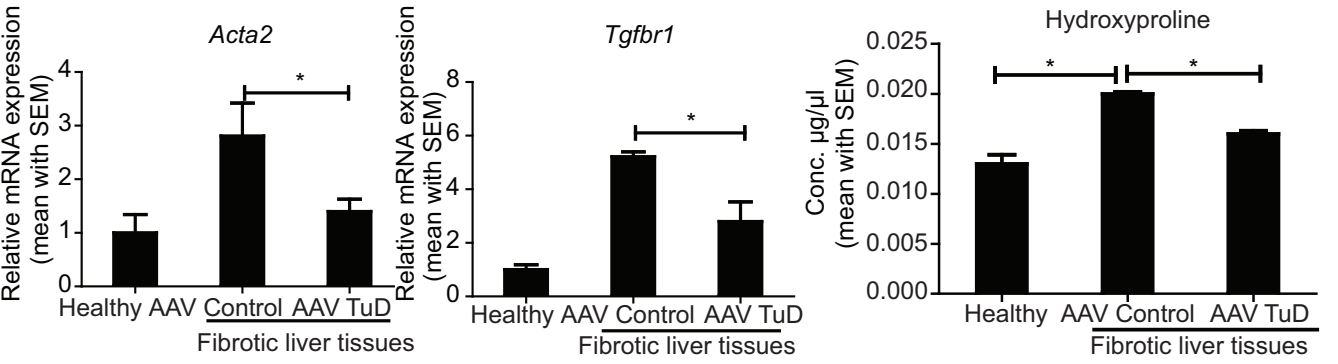

$\mathrm{H}$
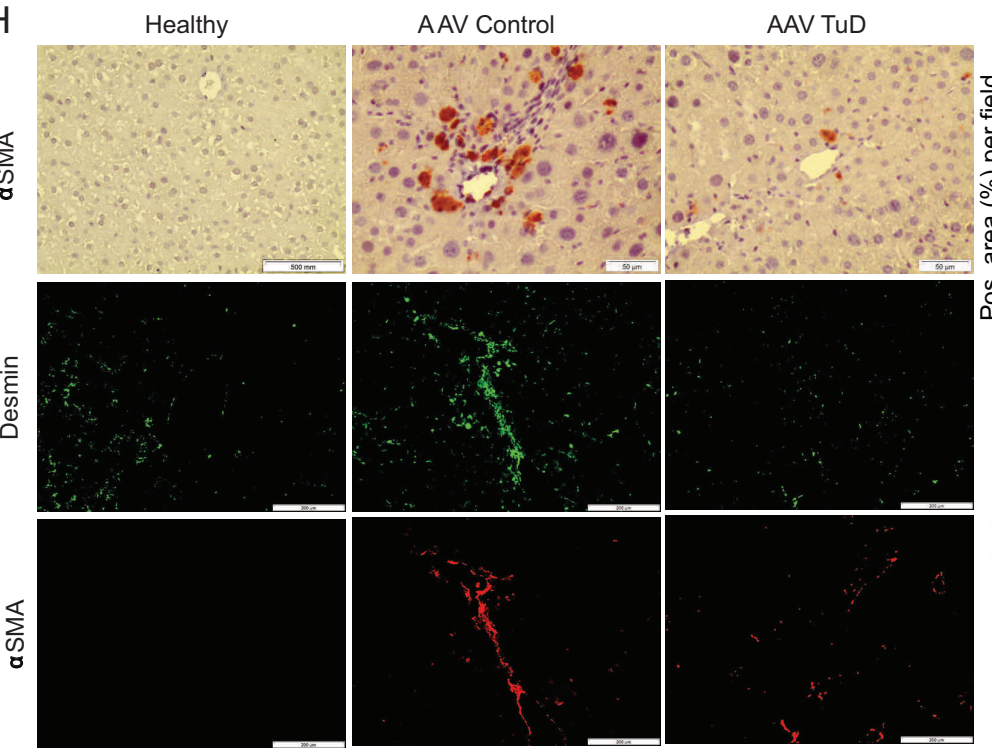

Fibrotic liver tissues
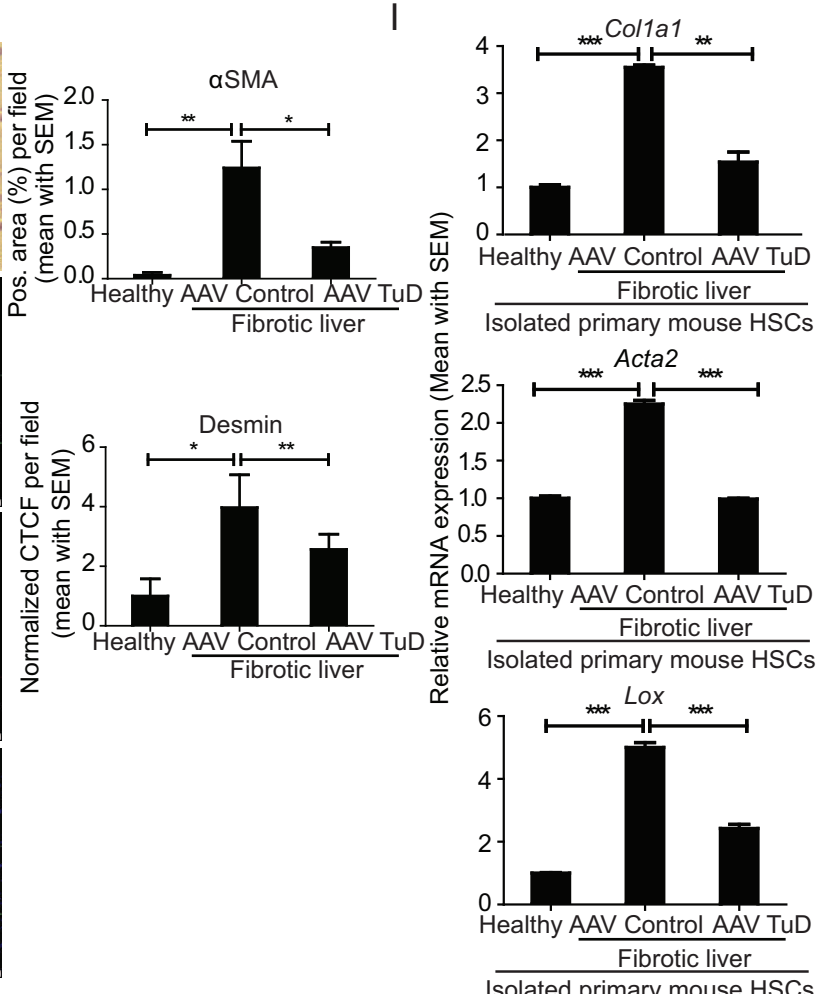
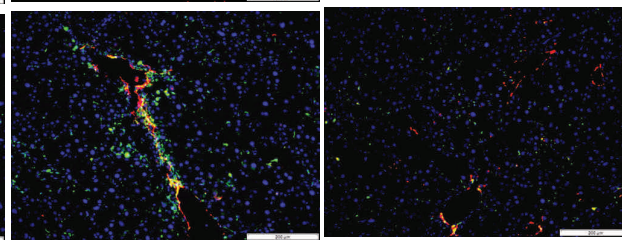

Figure 3 


\section{AFigure 4}

Position $814-820$ of 5 , ...GCUCCAUUAACCUAAAUGUAGCU... Gnai2 3'UTR

mmu-miR-221-3p

| | | | | |

3' CUUUgggucgucuguUACAUCGA

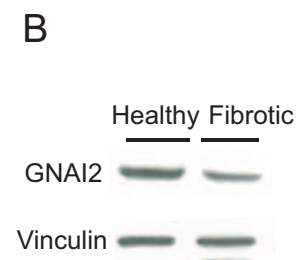

$\mathrm{D}$

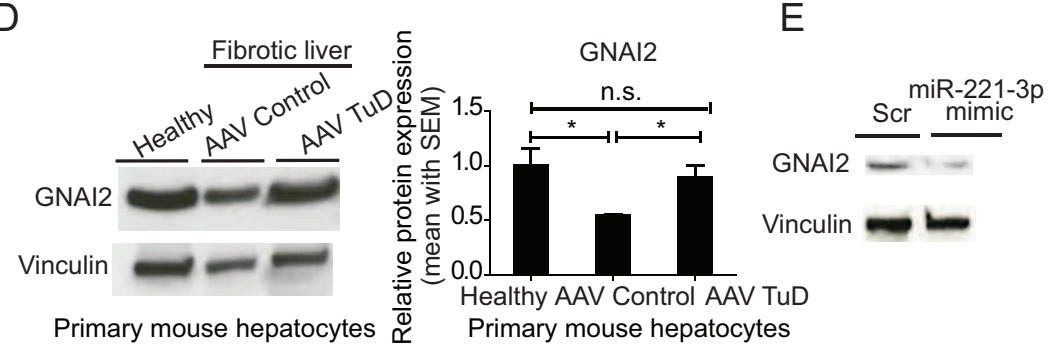

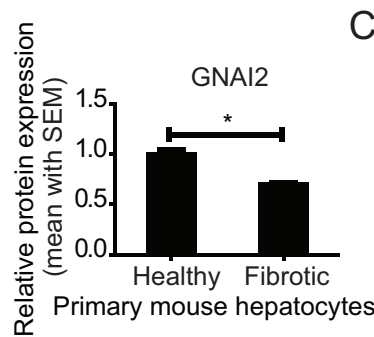

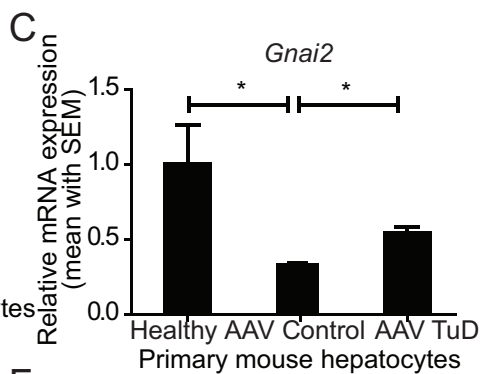

$\mathrm{F}$
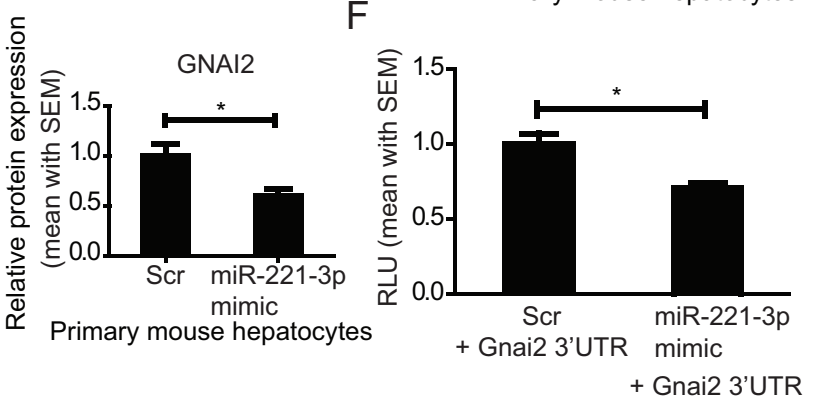

Primary mouse hepatocytes 


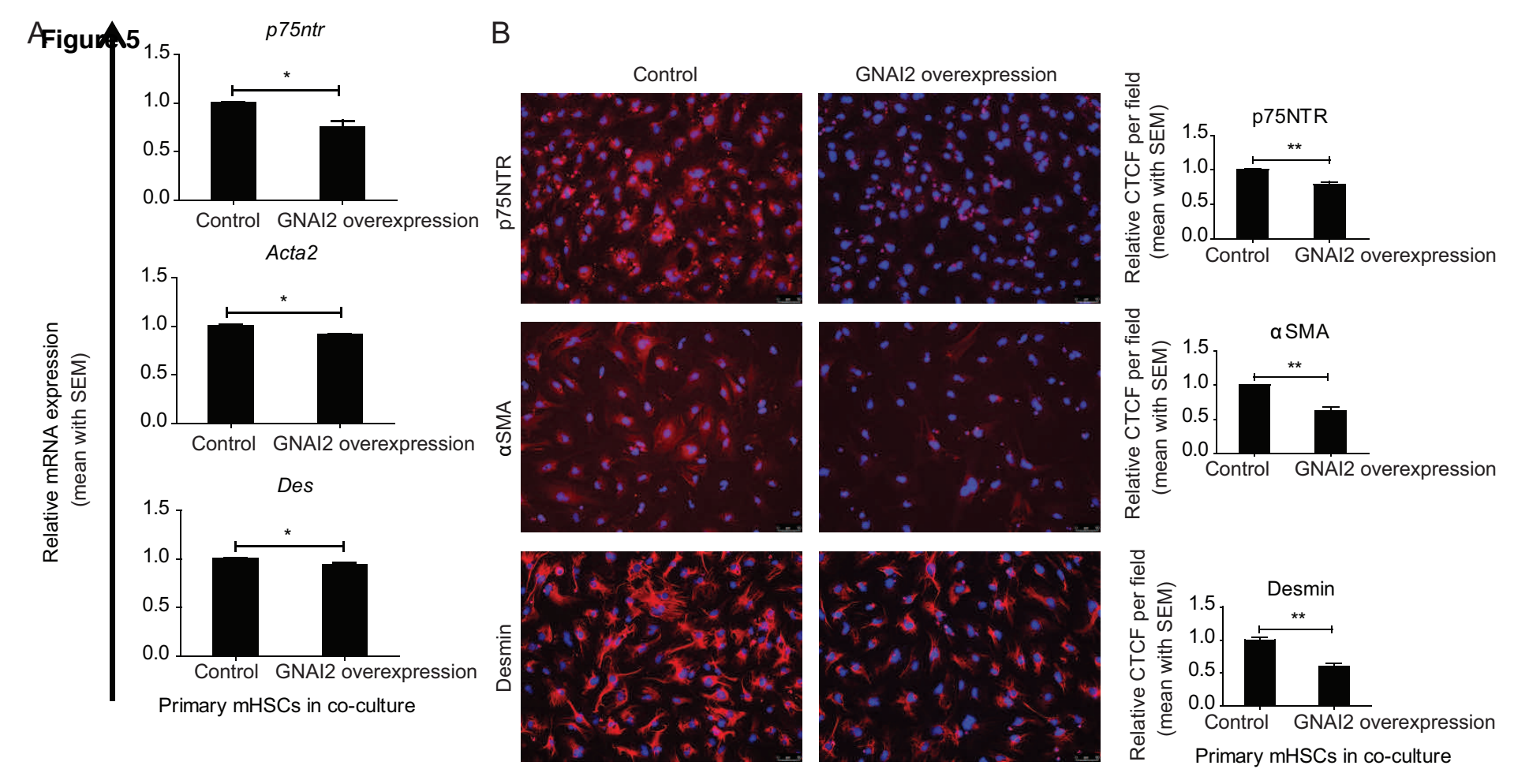

C
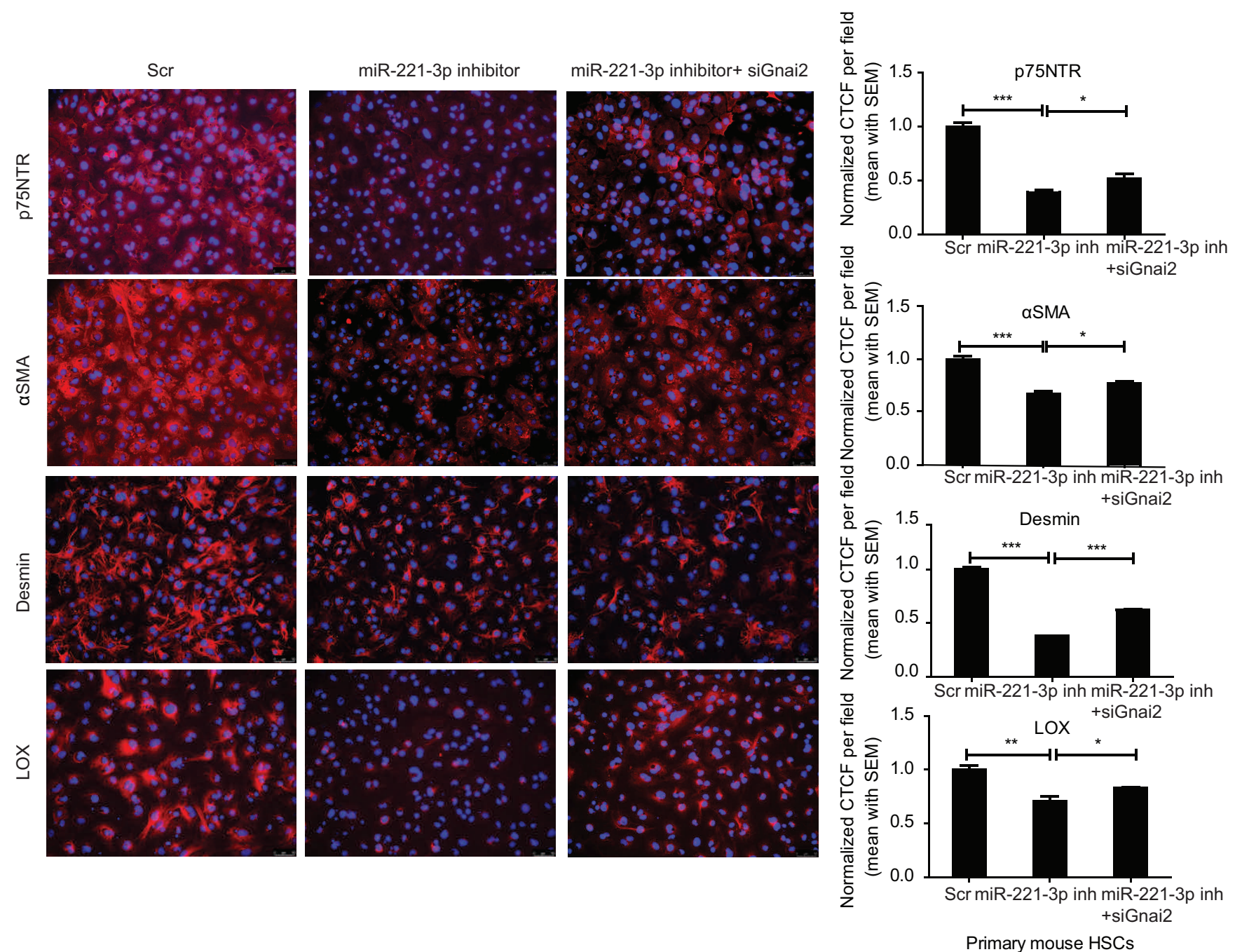

Figure 5 
Fiqure 6

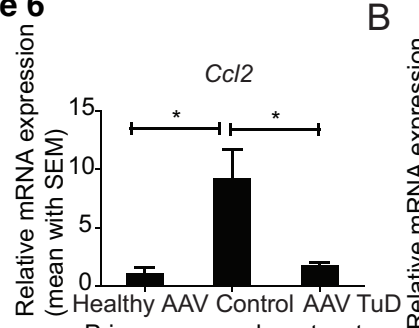

E

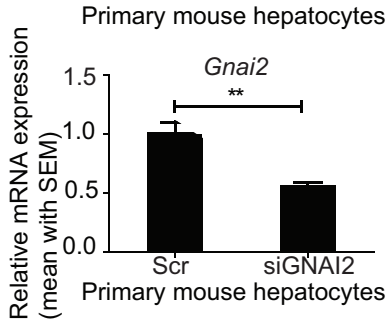

G
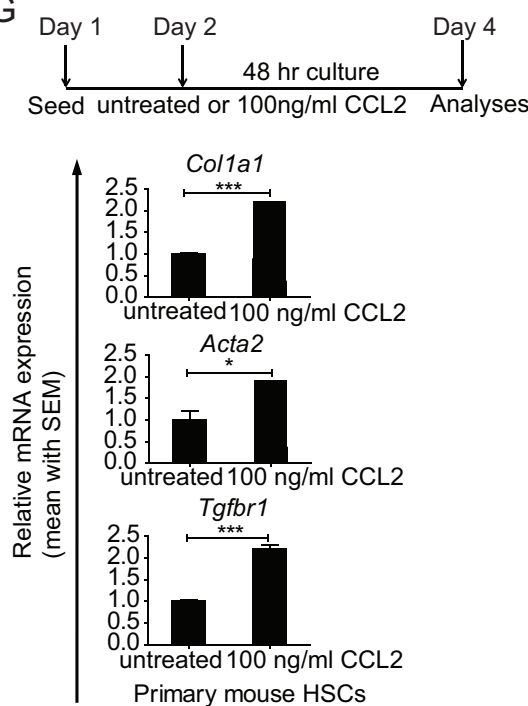

I Day 1 Day 2 Day $4 \quad$ Day 5 Day 7

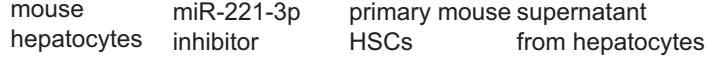

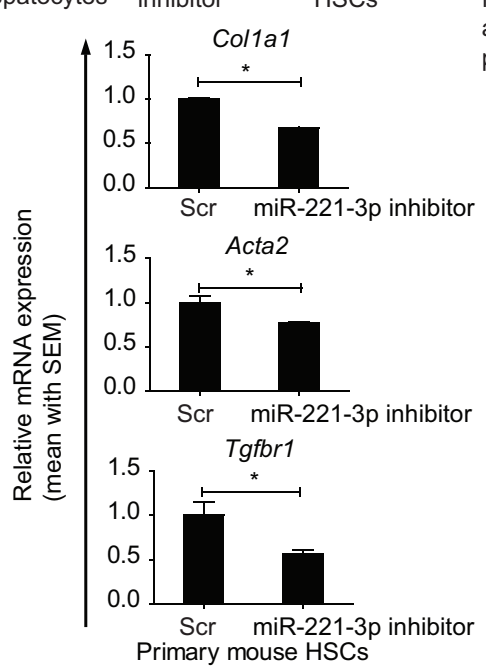

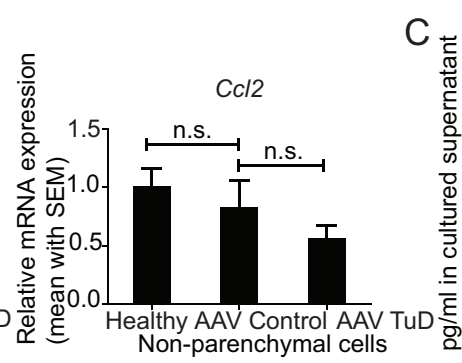

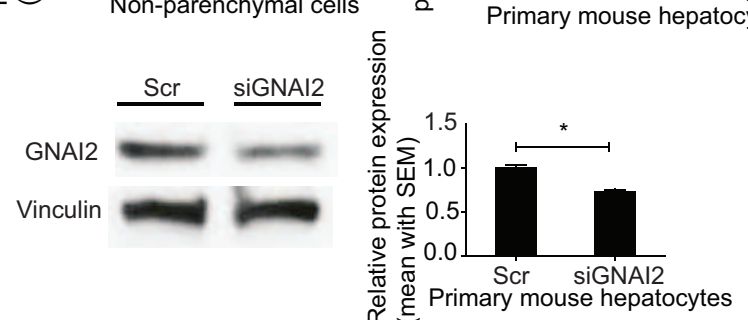

$\mathrm{H}$
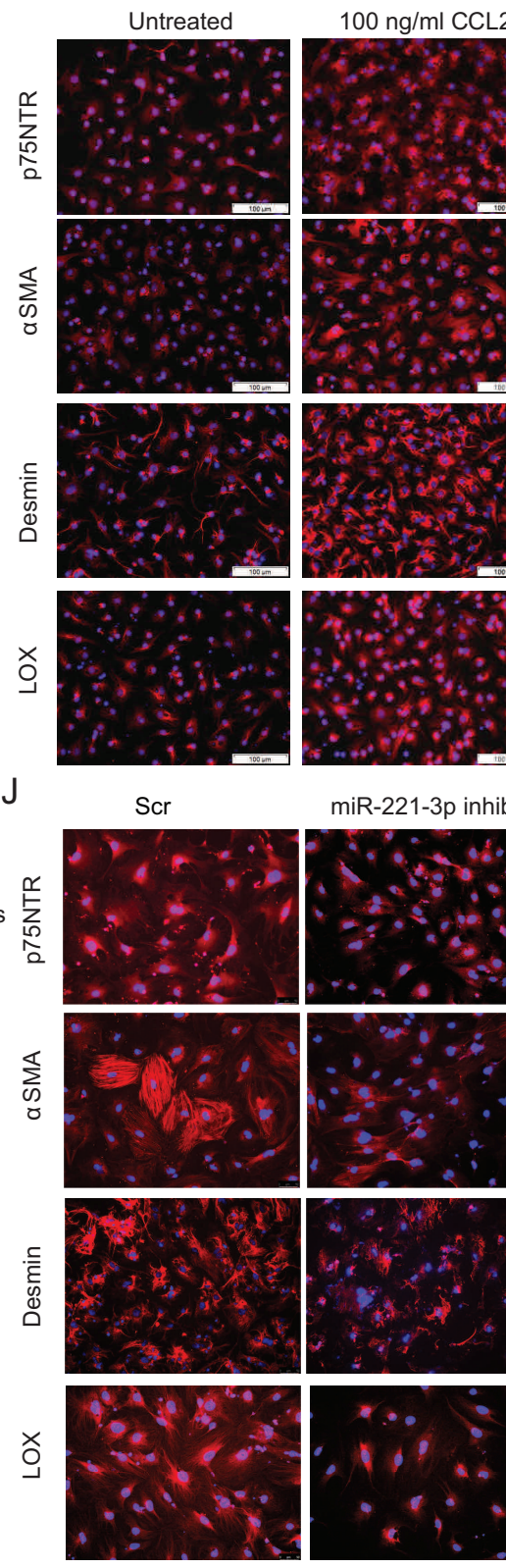

$100 \mathrm{ng} / \mathrm{ml} \mathrm{CCL} 2$
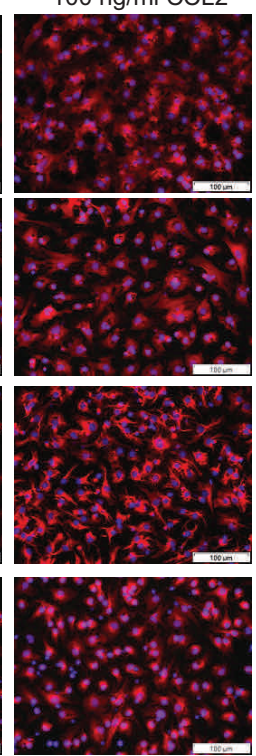

miR-221-3p inhibitor
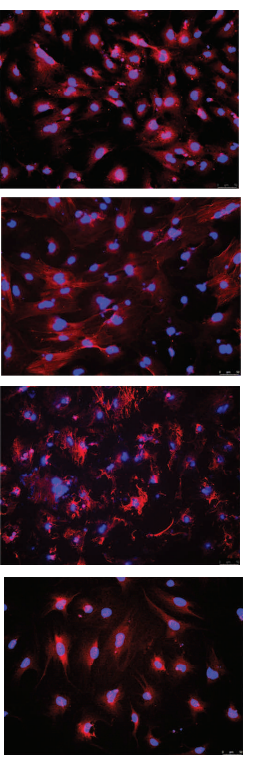

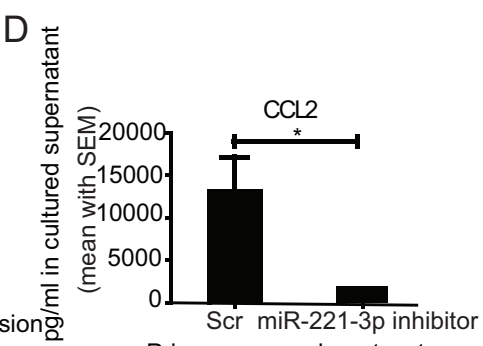

Primary mouse hepatocytes

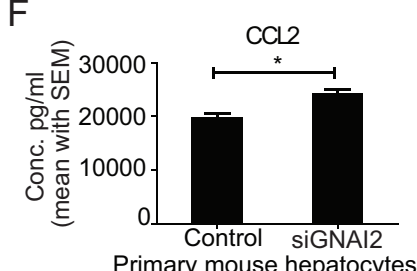

Primary mouse hepatocytes
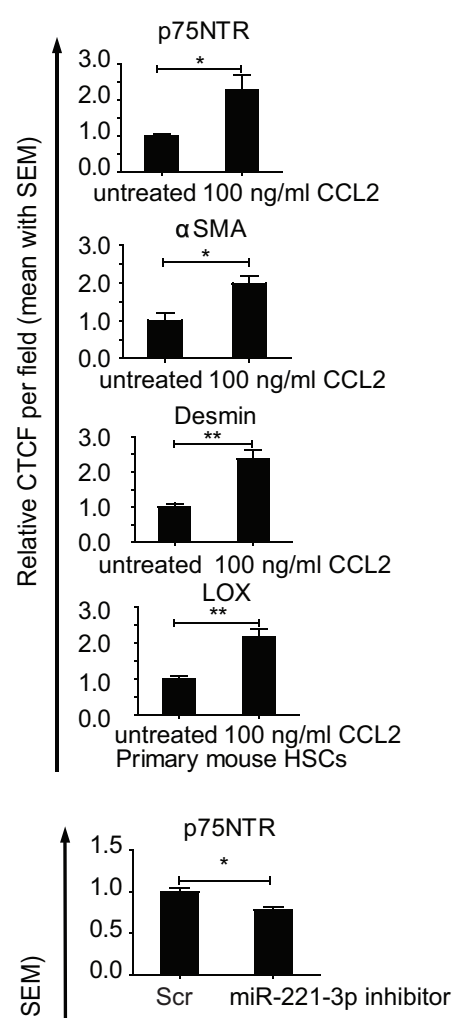
$15 \quad \alpha \mathrm{SMA}$
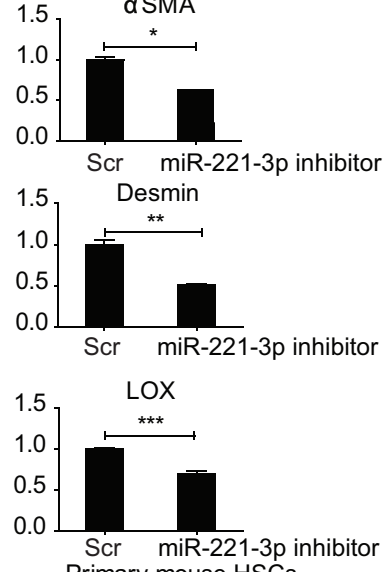

Figure 6 
Aigure 7

DDC 2 weeks

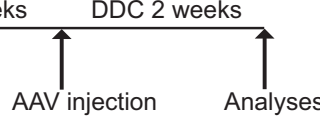

D
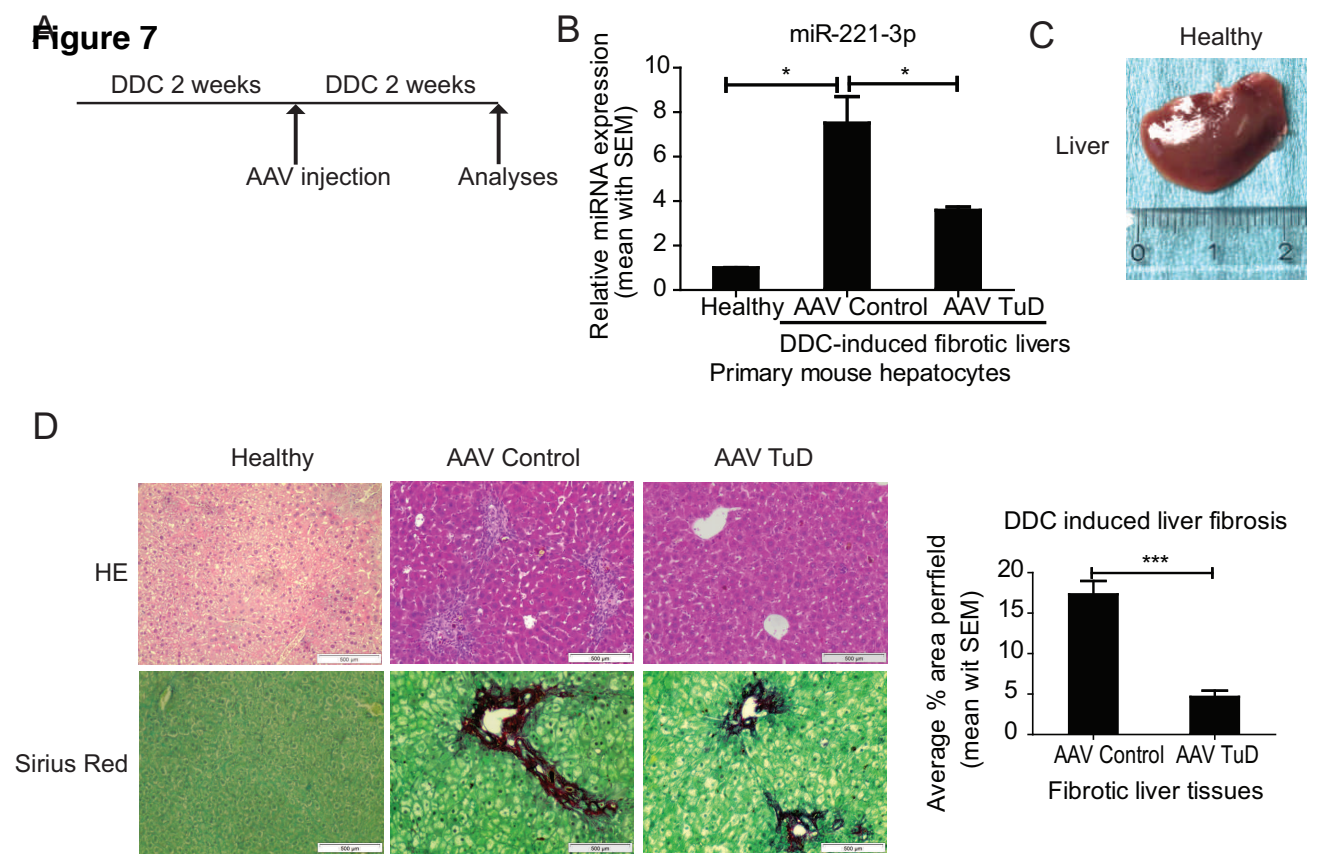

$\mathrm{F}$
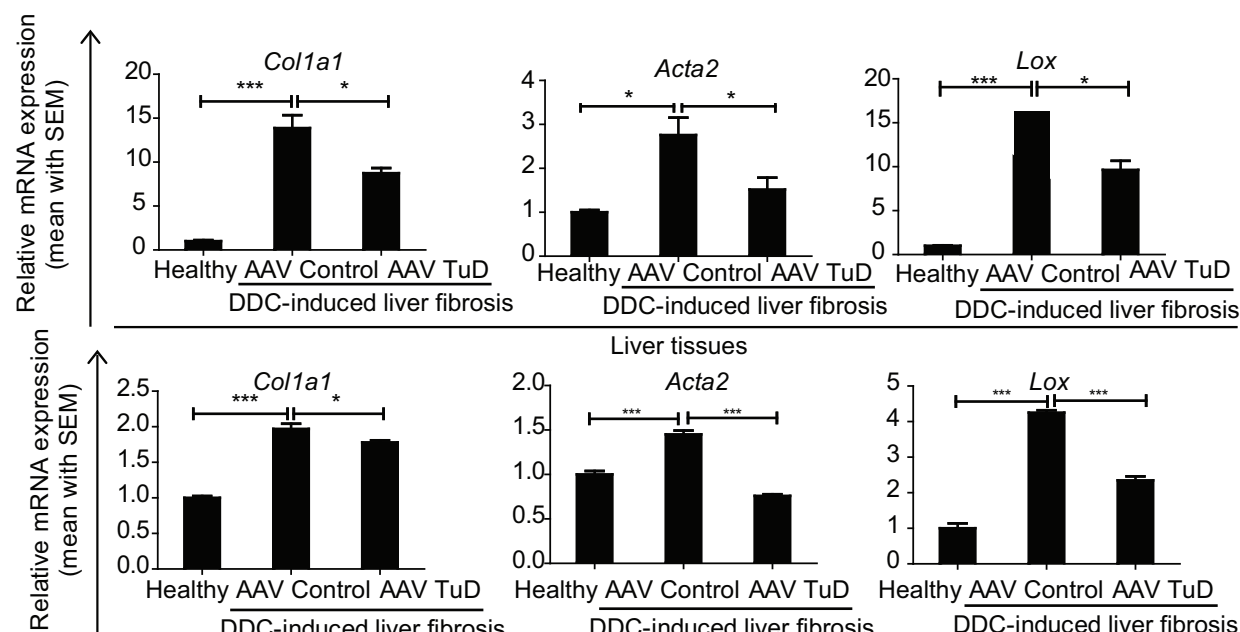

Healthy AAV Control AAV TuD Healthy AAV Control AAV TuD Primary mouse HSCs

G
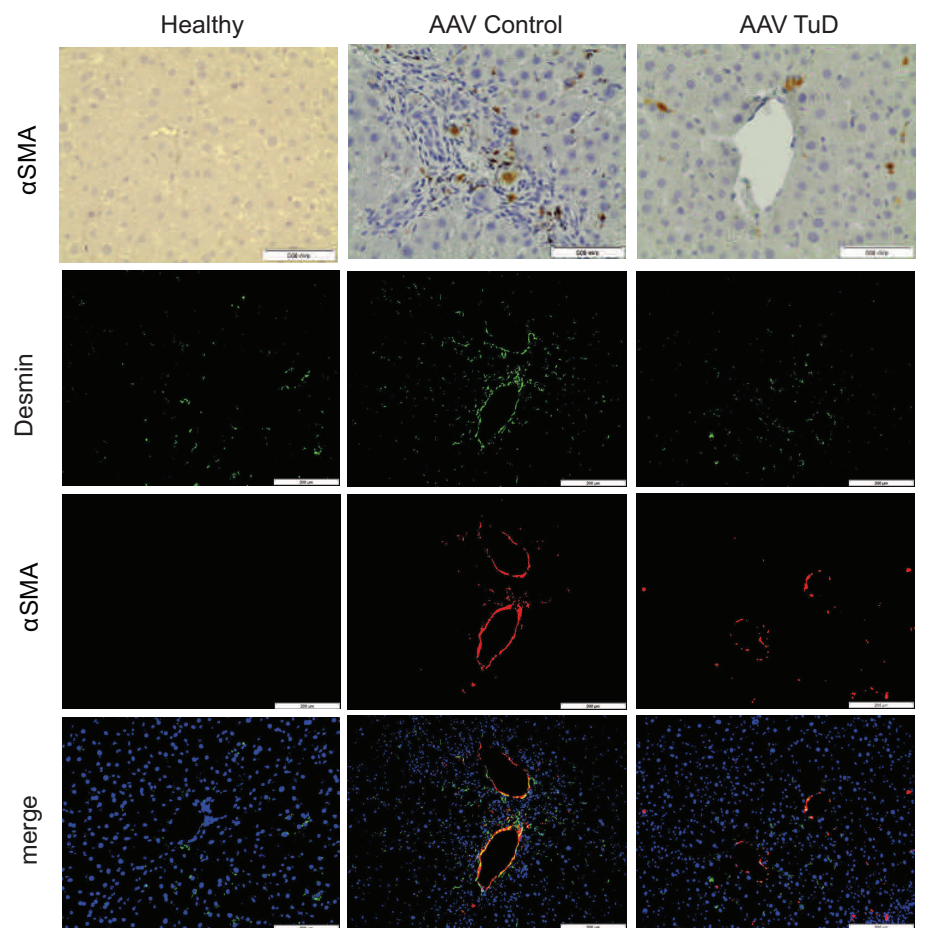
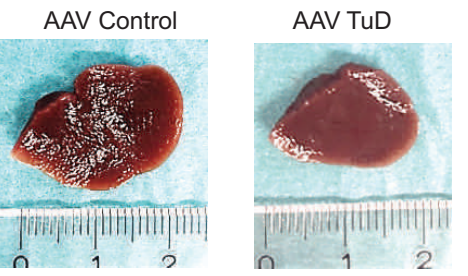

E

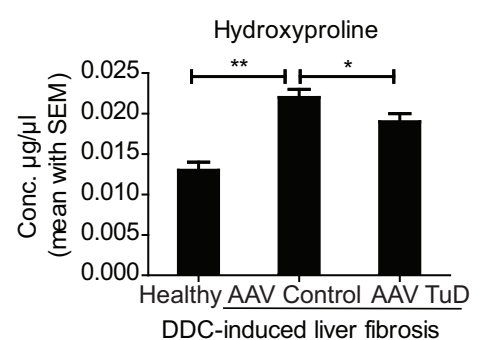

Figure 7 


\section{AFigure 8}

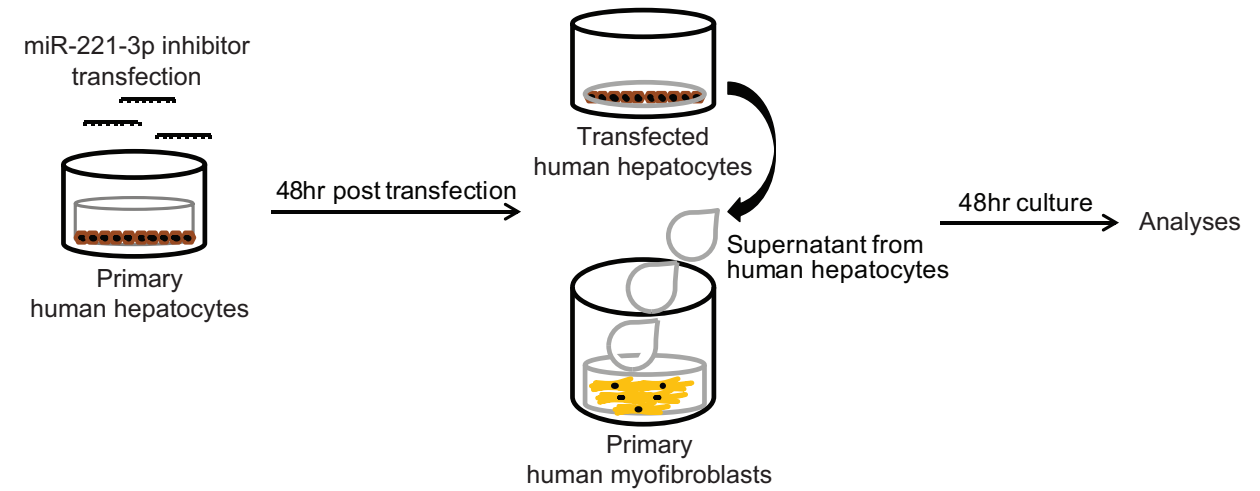

B

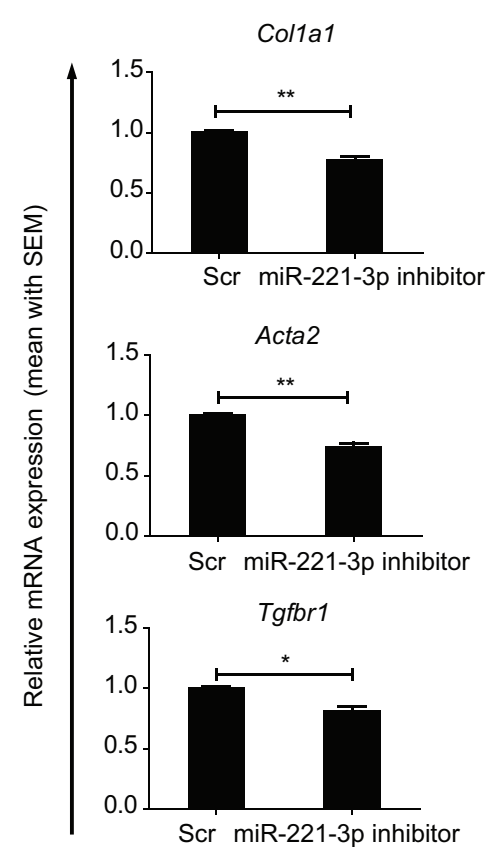

Primary human myofibroblasts
C

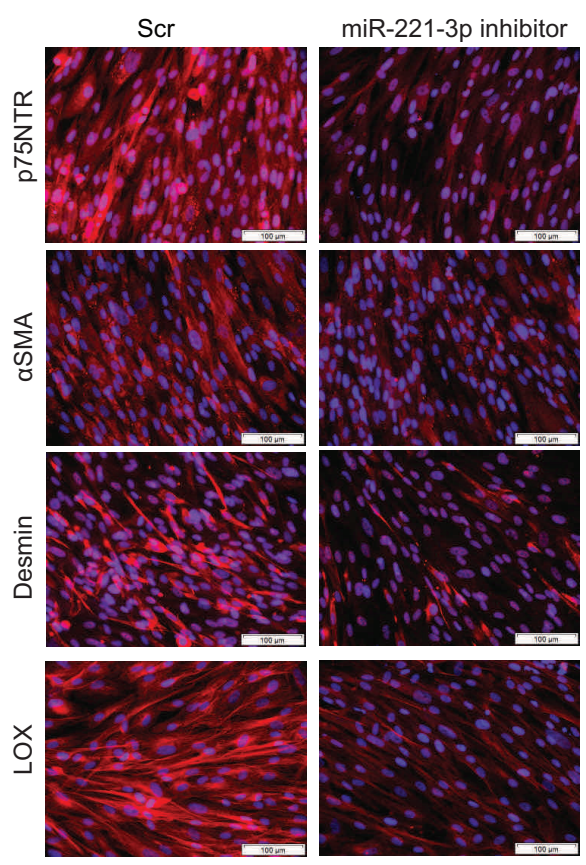

E

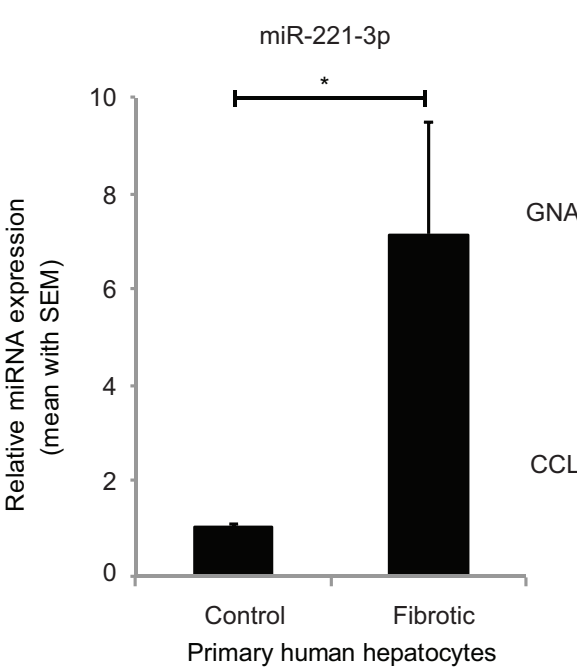

Primary human hepatocytes
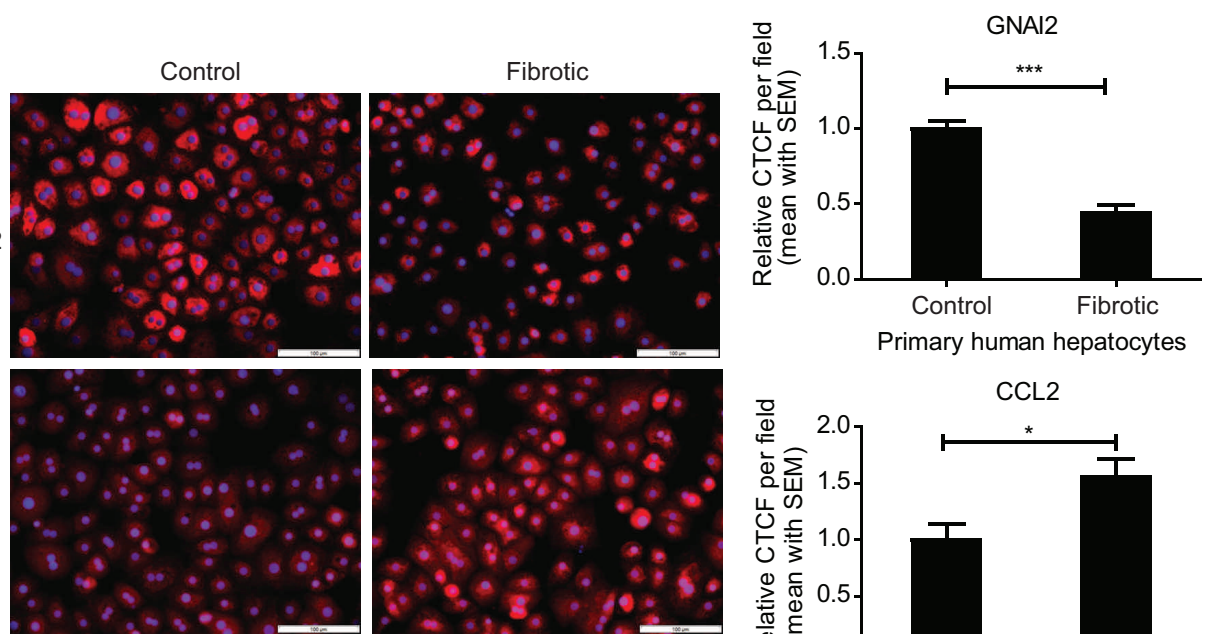

Fibrotic
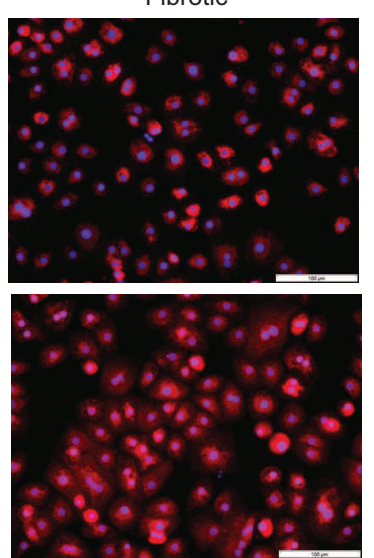

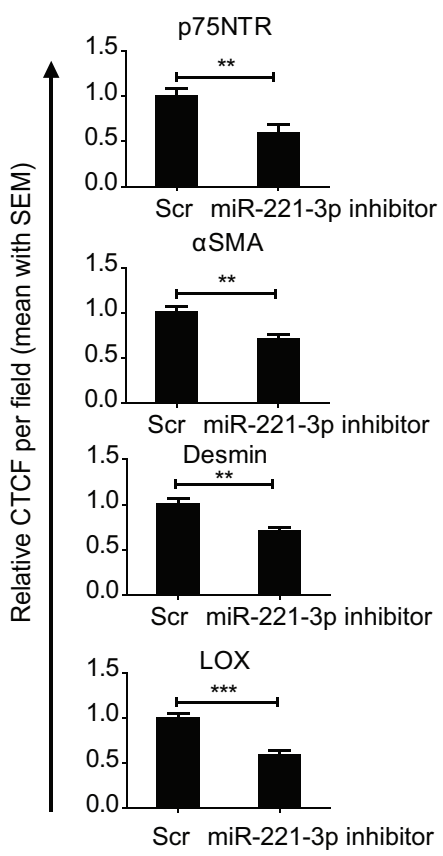

Primary human myofibroblasts

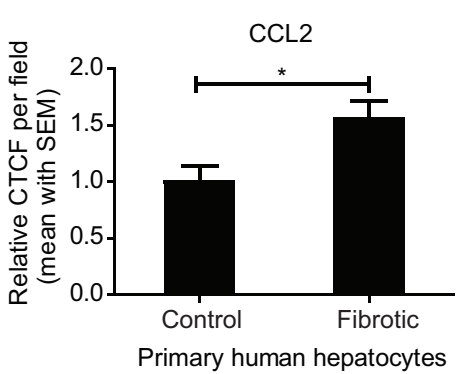

Figure 8 
Graphical Abstract

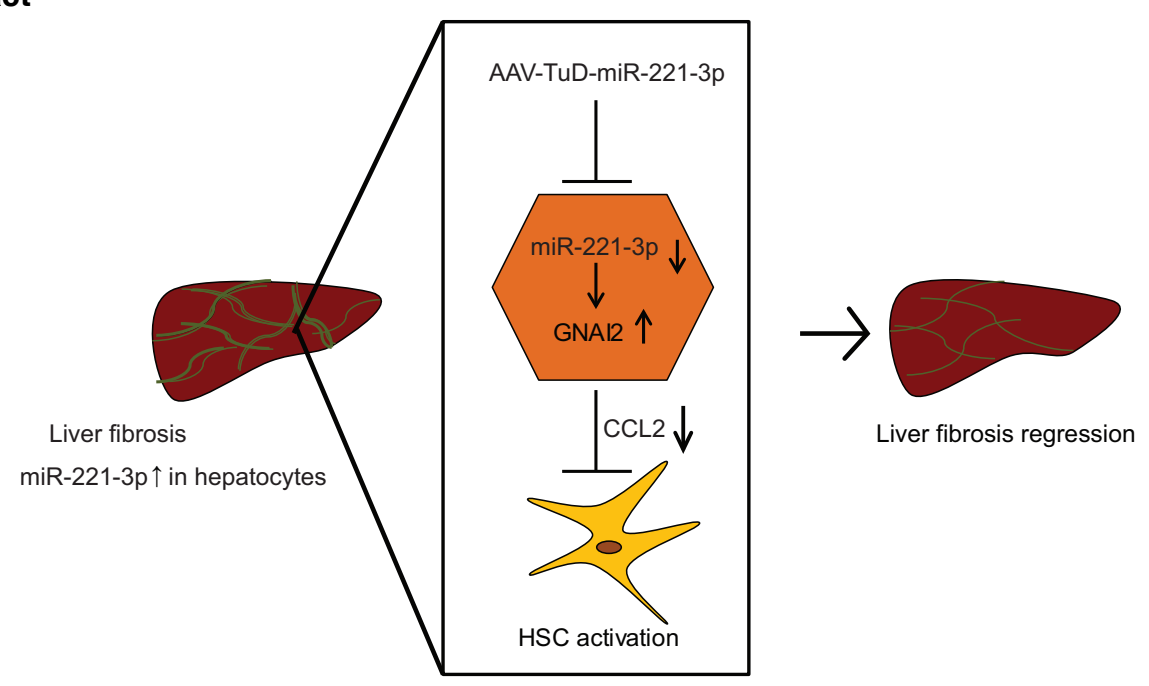

Graphical abstract 


\section{Supplementary data}

\section{Hepatocyte-specific suppression of microRNA-221-3p mitigates liver}

\section{fibrosis}

Hsin-Chieh Tsay ${ }^{1,2,3}$, Qinggong Yuan ${ }^{2,3}$, Asha Balakrishnan ${ }^{2,3}$, Marina Kaiser ${ }^{4}$, Selina Möbus ${ }^{1,2}$, Emilia Kozdrowska ${ }^{1,2}$, Marwa Farid ${ }^{1,2,3,5}$, Pia-Katharina Tegtmeyer $^{3,6}$, Katharina Borst ${ }^{3,6}$, Florian W.R. Vondran ${ }^{7,8}$, Ulrich Kalinke ${ }^{3,6}$, Andreas Kispert ${ }^{4}$, Michael P. Manns ${ }^{2}$, Michael Ott ${ }^{2,3}$, Amar Deep Sharma ${ }^{1,2}$

\section{Supplementary materials and methods}

Confirmation miRNA candidates in primary hepatocytes co-cultured with primary mouse HSCs

The 96-well inserts (Corning 3392) were first coated with $50 \mu \mathrm{g} / \mathrm{ml}$ collagen (Roche) for one hour at room temperature. The collagen solution was discarded and the wells were air-dried under cell culture hood. Each well was seeded with 7000 primary mouse hepatocytes in DMEM whole medium and after four hours the medium was changed to $80 \mu$ fresh $\mathrm{HCM}$ per well for overnight culture. Each miRNA mimic was transfected to primary mouse. Final 
concentration of each miRNA mimic was $25 \mathrm{nM}$. After 16 hours of transfection, the transfected primary mouse hepatocytes were placed on top of a monolayer of primary mouse HSCs (20000 cells per well) in DMEM whole medium for further 48 hour incubation at $37^{\circ} \mathrm{C}$. The supernatant was collected for further analyses.

Immunohistochemistry

Formalin-fixed, paraffin embedded liver tissues were cut into $2 \mu \mathrm{m}$ thick sections for immunohistochemical analyses with a standardized protocol. Following deparaffinization, antigen retrieval, and blocking, samples were incubated with aSMA antibody (1:200, Abcam, ab5694) at $4^{\circ} \mathrm{C}$, overnight. Samples were incubated with 1:500 of biotin-conjugated anti-goat secondary antibody (Vector Lab) at room temperature for one hour. AEC substrate (Dako) was used for color development. Hematoxylin was used as a counterstain. Samples were mounted with Faramount aqueous mounting medium (Dako).

Immunofluorescence staining Immunofluorescence staining was performed on $7 \mu \mathrm{m}$ cryosections using a standard protocol. Briefly, samples were blocked with $5 \%$ serum in PBS at 
room temperature for one hour and incubated overnight, at $4^{\circ} \mathrm{C}$, with the primary antibodies. The samples were incubated with 1:500 of the secondary antibody (Alexa Fluor 594, Invitrogen, A21207), at room temperature for 30 minutes. Nuclei were stained with DAPI (Sigma). The primary antibodies were p75NGF (1:100, Abcam, ab8874) and desmin (1:100, Thermo Scientific, RB-9014-P0). For co-staining, cryosection slides were first thawed at room temperature for 20 minutes and washed with PBS for five minutes. The slides were blocked with the blocking solution ( $5 \%$ donkey serum and $0.3 \%$ Triton $X$ in PBS, use $0.1 \%$ Triton $X$ when staining with $\mathrm{p} 75 \mathrm{NTR}$ ) at room temperature for 30 minutes. The slides were later incubated with the mixture of two primary antibodies (desmin and p75NTR or desmin and aSMA, 1:100 dilution each antibody) in the blocking solution at $4^{\circ} \mathrm{C}$ overnight. Wash three times with PBS for five minutes each and incubate the slides with the mixture of two secondary antibodies (Alexa 594 donkey anti rabbit and Alexa 488 donkey anti goat, 1:500 dilution each) for 30 minutes at room temperature. Wash three times with PBS for five minutes each and stain the slides with Hoechast (1mM stock solution, 1:50 dilution) for 10 minutes. Wash three times with PBS for five minutes each and mount the slides with Dako Faramount aqueous mounting medium. 
Total RNA isolation and quantitative real-time PCR for miRNA expression

Total RNA from cell pellets or liver tissues was isolated with Qiazol (Qiagen) and treated with DNase (Ambion) according to the manufacturer's instructions. $50 \mathrm{ng}$ of total RNA were reversely transcribed to cDNA (Applied Biosystems). $1 \mu \mathrm{l}$ of 1:10 diluted cDNA was used per qPCR reaction. The TaqMan qPCR $2 X$ master mix (ThermoFisher Scientific) was used. Relative miRNA expression levels were normalized to the small nuclear RNA, U6. The second derivative maximum method was used for quantification. All qPCRs were performed on the LightCycler 480 II (Roche).

Luciferase reporter assay

The 3' UTR of Gnai2 was amplified by PCR from genomic DNA and was cloned into a miRGLO vector (Promega). The miRGLO-Gnai2 vectors were co-transfected with miR-221-3p mimic or scramble into primary hepatocytes. Luciferase activity was measured with the Dual-Glo Luciferase Assay (Promega), 48 hours after transfection. The procedure was followed according to the manufacturer's instructions. 
Immunoblotting

Protein samples were separated by $4-12 \%$ TruPAGE Precast gel (Sigma) and were later transferred to PVDF membranes (Millipore). Membranes were blocked with $5 \%$ milk-TBST at room temperature for one hour. Membranes were incubated with primary antibodies at $4{ }^{\circ} \mathrm{C}$ overnight and then incubated with HRP-conjugated secondary antibodies at room temperature for one hour. ECL substrate (Thermo Fisher Scientific) was applied on membranes at room temperature for two minutes. The exposure time ranged from 30 seconds to 10 minutes depending on the protein of interest. The primary antibodies used were GNAI2 (Abcam, ab137050) 1:1000; PUMA (Abcam, ab9643) 1:1000; Vinculin (Sigma, V9131-100UL) 1:2000.

Co-culture of primary mouse hepatocytes and primary mouse HSCs 100,000 primary hepatocytes were seeded per transwell insert (Corning, 3494) for overnight culture. Hepatocytes were then transfected with either $1 \mu \mathrm{g}$ control vector (pCMV-GFP) or $1 \mu \mathrm{g}$ GNAI2 expression vector (pCMV-GNAI2-GFP, Biocat). After 12 to 16 hours of transfection, the insert was placed on the top of primary mouse HSCs $(200,000$ cells per well) for further 48 hours co-culture. The primary moue HSCs were fixed with $4 \%$ PFA 
or the cell pellets were collected for further experiments.

Primary mouse HSCs culture treated with CCL2

100,000 primary HSCs were seeded per well in 24-well plates one day before

CCL2 treatment. The HSCs were treated with or without $100 \mathrm{ng} / \mathrm{ml}$ of CCL2 (Abcam, ab9901) for 48 hours. The supernatant and cell pellets were collected for further analyses.

Primary human myofibroblasts treated with the supernatant collected from miR-221-3p inhibitor treated primary human hepatocytes 250,000 primary human hepatocytes were seeded per well in 12 -well plates one day before the miR-221-3p inhibitor transfection. Four hours after seeding, the medium was changed to fresh HCM. Virofect-enhanced hepatocytes were transfected with $25 \mathrm{nM}$ miRNA inhibitors or scramble control with TargeFect F-2 (Targeting system). The supernatant was collected after 48 hours of post transfection and was applied on primary human myofibroblasts for further culture. The primary myofibroblasts were fixed or collected after 48-hour culture. 
CCL2 detection by ELISA

The supernatant was collected from primary mouse hepatocytes transfected with Gnai2 cDNA vectors or miR-221 inhibitors for 48 hours post transfection. The supernatant was diluted from $1: 4$ to $1: 50$ to acquire reliable signals. The procedure of detecting mouse CCL2 in supernatant followed the manufacturer's instruction from the Mouse CCL2 ELISA Ready-SET-Go kit (eBioscience). 


\section{Supplementary figures:}

\section{Figure S1}

A

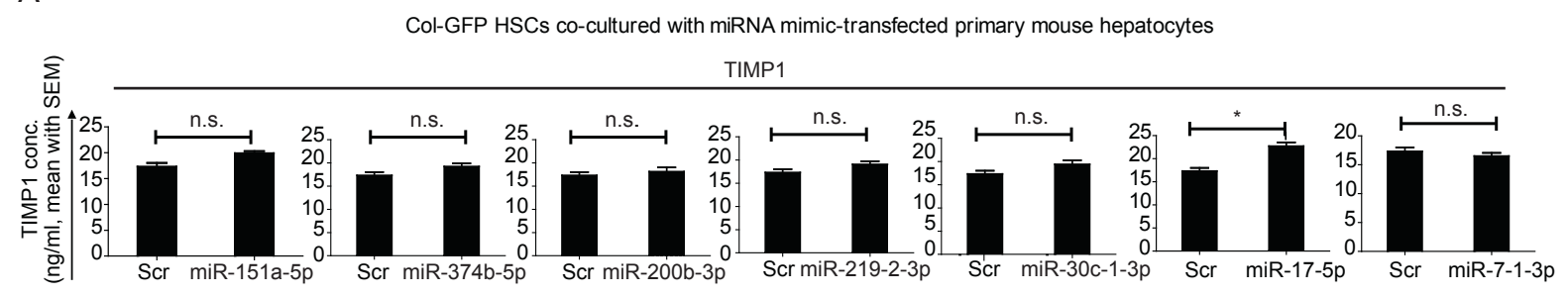

B Primary mouse HSCs co-cultured with miR-17-5p mimic-transfected primary mouse hepatocytes

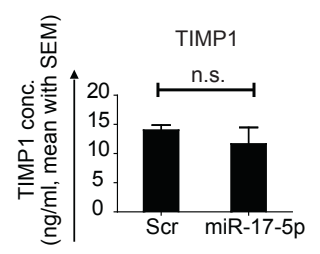

C

Primary mouse HSCs co-cultured with miR-221-3p mimic-transfected primary mouse hepatocytes

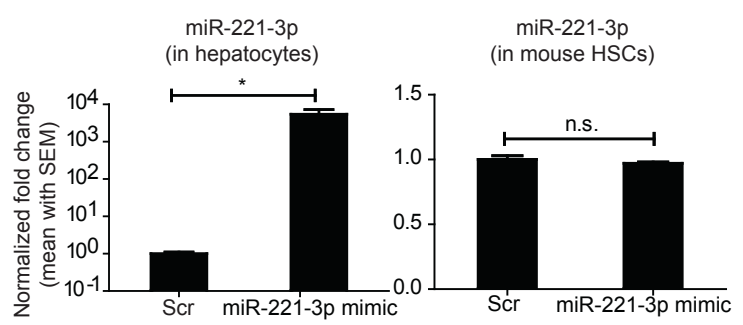

Supplementary Fig. S1. Identification of miR-221-3p as a candidate miRNA. (A) TIMP1 ELISA showed only transfection of miR-17-5p in addition to miR-221-3p significantly increased TIMP1 levels when miRNAs were transfected into primary mouse hepatocytes and subsequently co-cultured with Col-GFP HSCs. (B) TIMP1 ELISA revealed unchanged TIMP1 levels when miR-17-5p was transfected into primary mouse hepatocytes and subsequently co-cultured with primary mouse HSCs. (C) The q-PCR analyses show that only miR-221-3p transfected hepatocytes had significant upregulation of 
miR-221-3p (left) but not co-cultured primary mouse HSCs (right). Error bars represent \pm SEM. One-way ANOVA was used for statistical evaluation. $\left({ }^{*} p<\right.$ 0.05 , n.s. not significant $)(n=4)$.

\section{Figure S2}
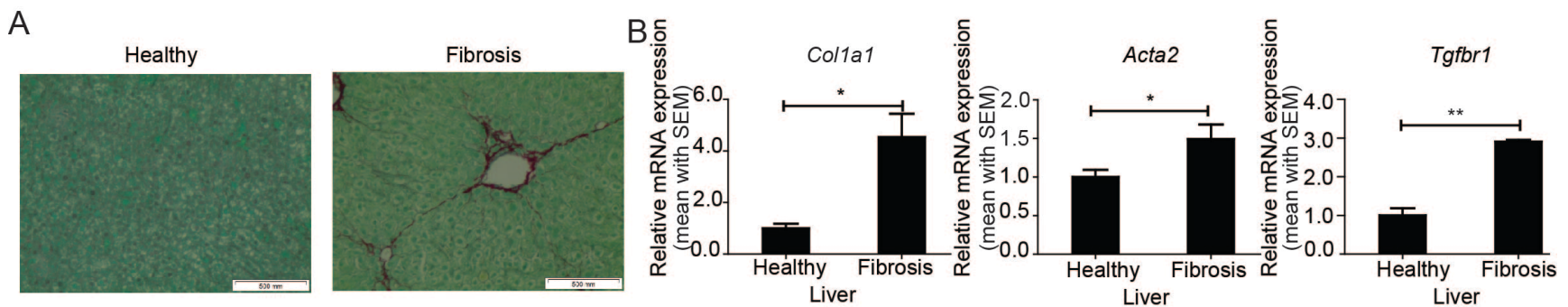

Supplementary Fig. S2. Validation of a $\mathrm{CCl}_{4}$-induced liver fibrosis model.

(A) Collagen deposition shown in liver fibrosis by Sirius red staining. (B) Upregulation of fibrotic markers, Col1a1, Acta2 and Tgfbr1 in fibrotic livers. Magnification: 100X. Error bars represent \pm SEM. One-way ANOVA was used for statistical evaluation. $\left({ }^{*} p<0.05 ;{ }^{* *} p<0.01\right)(n=3)$. 


\section{Figure S3}

A
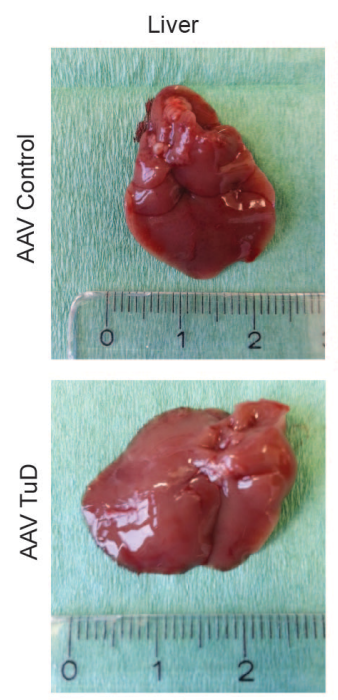

B

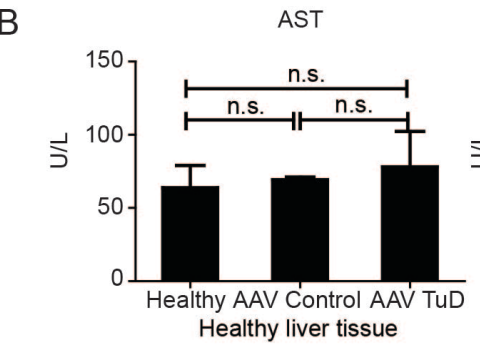

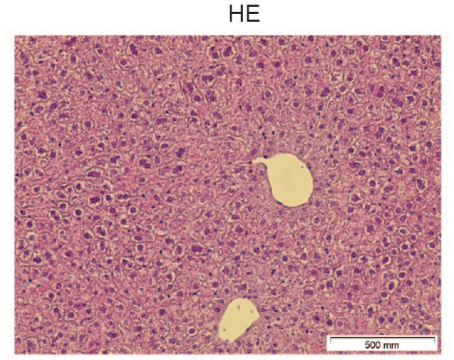

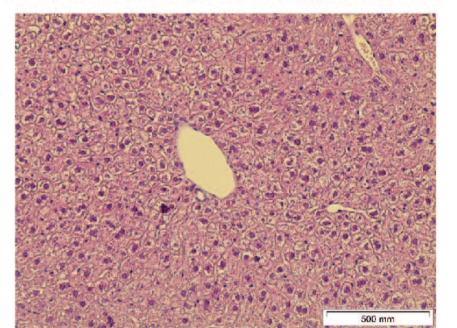

ALT
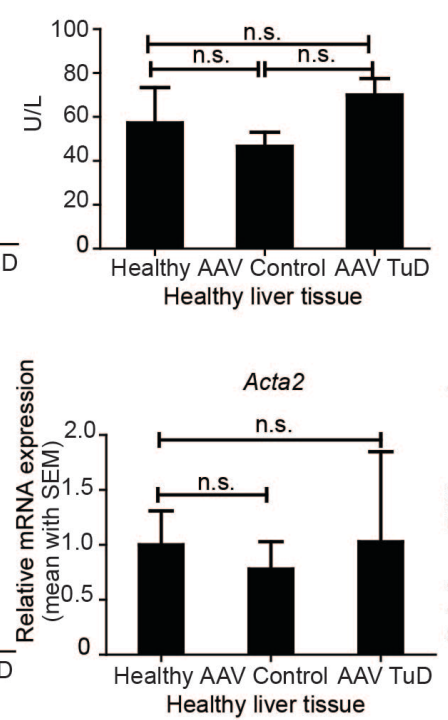

Sirius Red
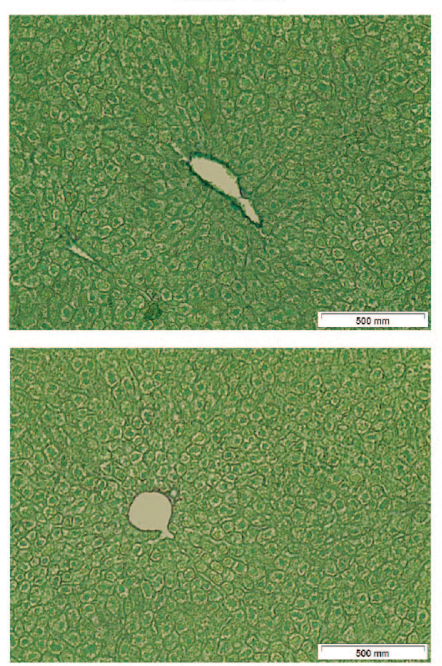

Bilirubin
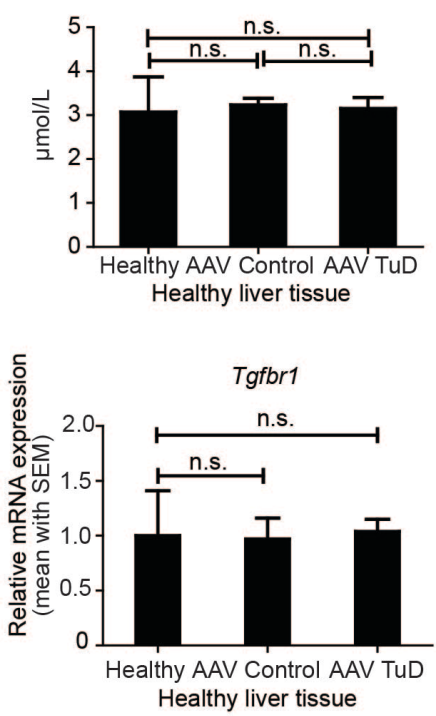

Supplementary Fig. S3. AAV TuD did not induce liver fibrosis in uninjured mice. (A) Lack of micronodules (gross morphology), cellular infiltration (HE staining), and liver fibrosis (Sirius Red staining) in AAV Control or AAV TuD injected healthy mice. (B) Comparable levels of AST, ALT, and bilirubin in serum from healthy BALB/c mice and healthy BALB/c mice injected with AAV control or AAV TuD. (C) Comparable mRNA expression levels of 
Col1a1, Acta2, and Tgfbr1 in liver tissues from healthy BALB/c mice and healthy BALB/c mice injected with AAV Control or AAV TuD. Magnification: 100X. Error bars represent \pm SEM. One-way ANOVA was used for statistical evaluation. (n.s. not significant) $(n=3)$.

\section{Figure S4}

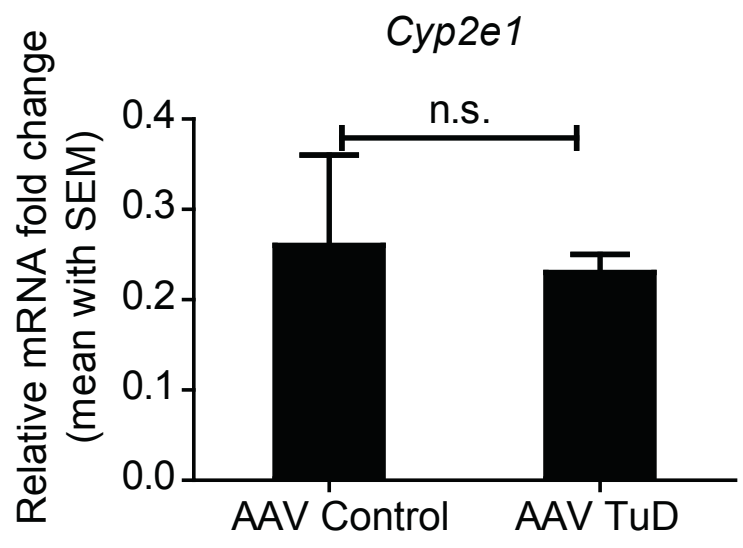

Primary mouse hepatocytes from fibrotic liver tissues

Supplementary Fig. S4. Analysis of Cyp2e1 expression in hepatocytes from mice injected with AAV Control or AAV TuD. Q-PCR results showed unchanged Cyp2e1 mRNA expression in livers of mice injected either with AAV Control or AAV TuD injected mice ( $n=7$ mice/group). Error bars represent 土 SEM. Two-tailed student's T-test was used for statistical evaluation. (n.s. not significant). 


\section{Figure S5}

A B
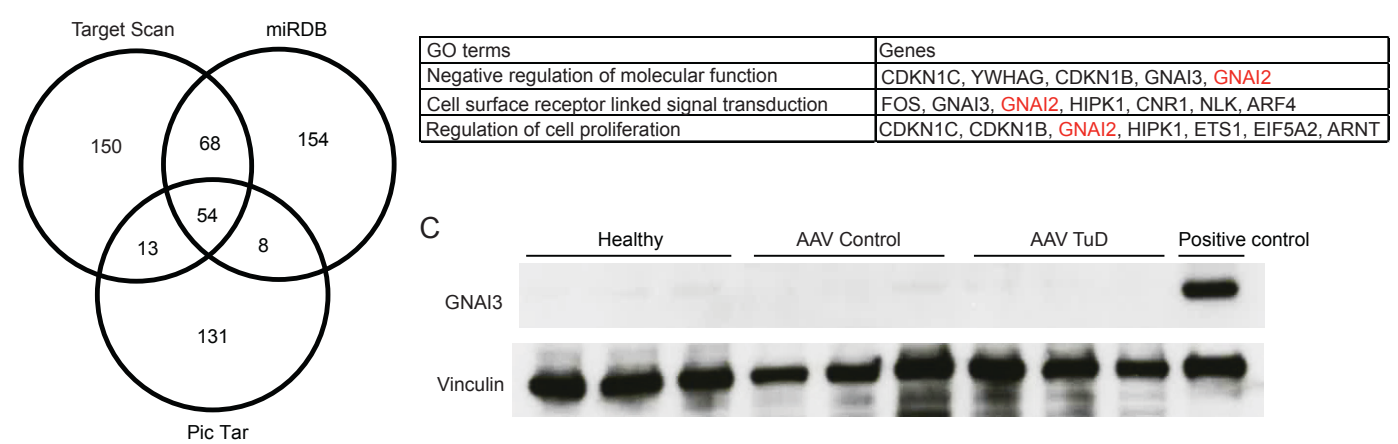

Supplementary Fig. S5. Gnai2 is a potential target gene of miR-221-3p. (A) Venn diagram showing 54 potential miR-221-3p target genes from three different miRNA target prediction tools. (B) Gnai2 was selected based on gene ontology terms (GO). (C) No significant regulation of GNAI3 in primary mouse hepatocytes upon miR-221-3p inhibition in vivo $(n=3)$.

Figure S6
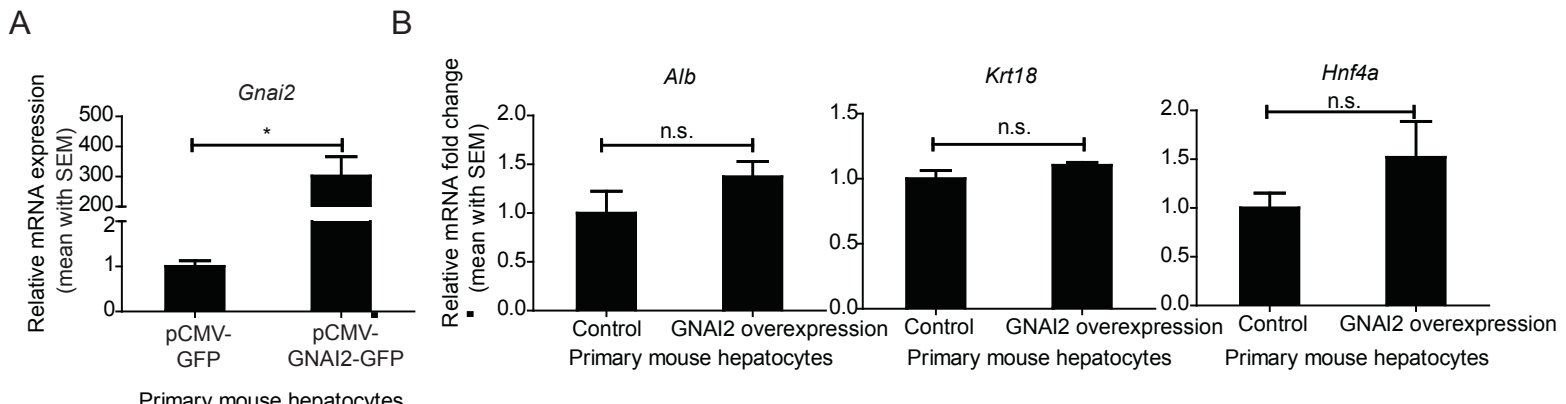

Supplementary Fig. S6. Transient GNAl2 overexpression with

GNAI2-expressing plasmid in primary mouse hepatocytes. (A) Q-PCR 
results show overexpression of Gnai2 mRNA in primary mouse hepatocytes transfected with Gnai2 overexpressing cDNA plasmid. (B) Q-PCR analyses for hepatocyte markers such as Alb, Krt18 and Hnf4a upon Gnai2 overexpression compared to control. Error bars represent \pm SEM. Two-tailed student's T-test was used for statistical evaluation. $\left({ }^{*} p<0.05\right)(n=3)$.

\section{Figure S7}

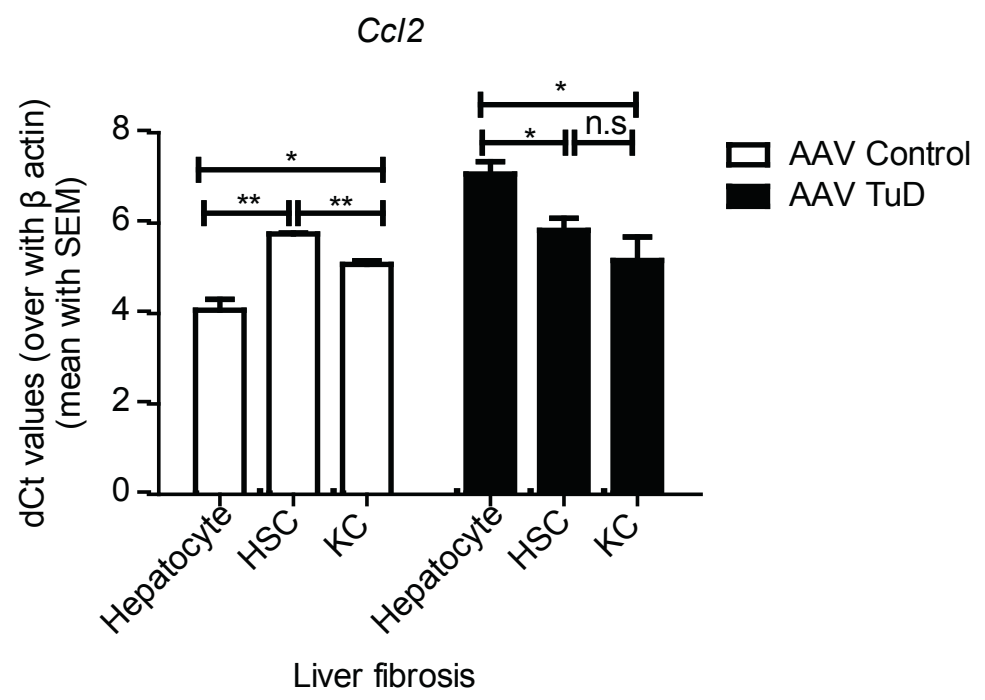

Supplementary Fig. S7. Analysis of $\mathrm{Cc/2}$ expression in various hepatic cell types from fibrotic liver. Q-PCR results showed as dCt values, which were normalized with $\beta$ actin in hepatocytes, HSCs, and Kupffer cells from mice injected either with AAV control or AAV TuD $(n=3)$. Error bars represent \pm SEM. One-way ANOVA was used for statistical evaluation. $\quad\left({ }^{*} p<0.05\right.$; $\left.{ }^{* *} \mathrm{p}<0.01\right)$ 


\section{Figure S8}

A
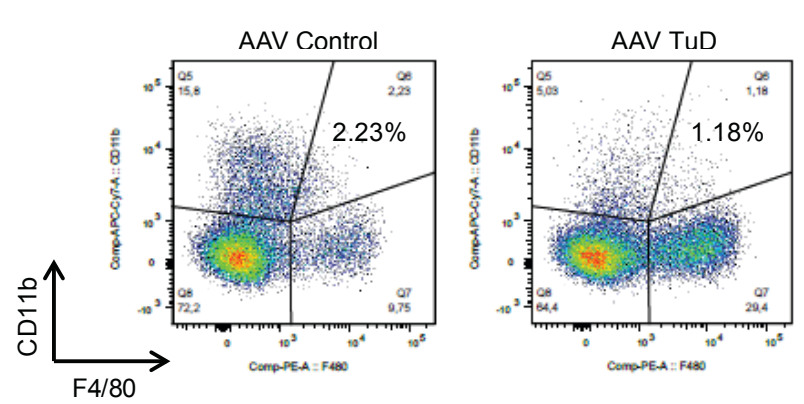

B
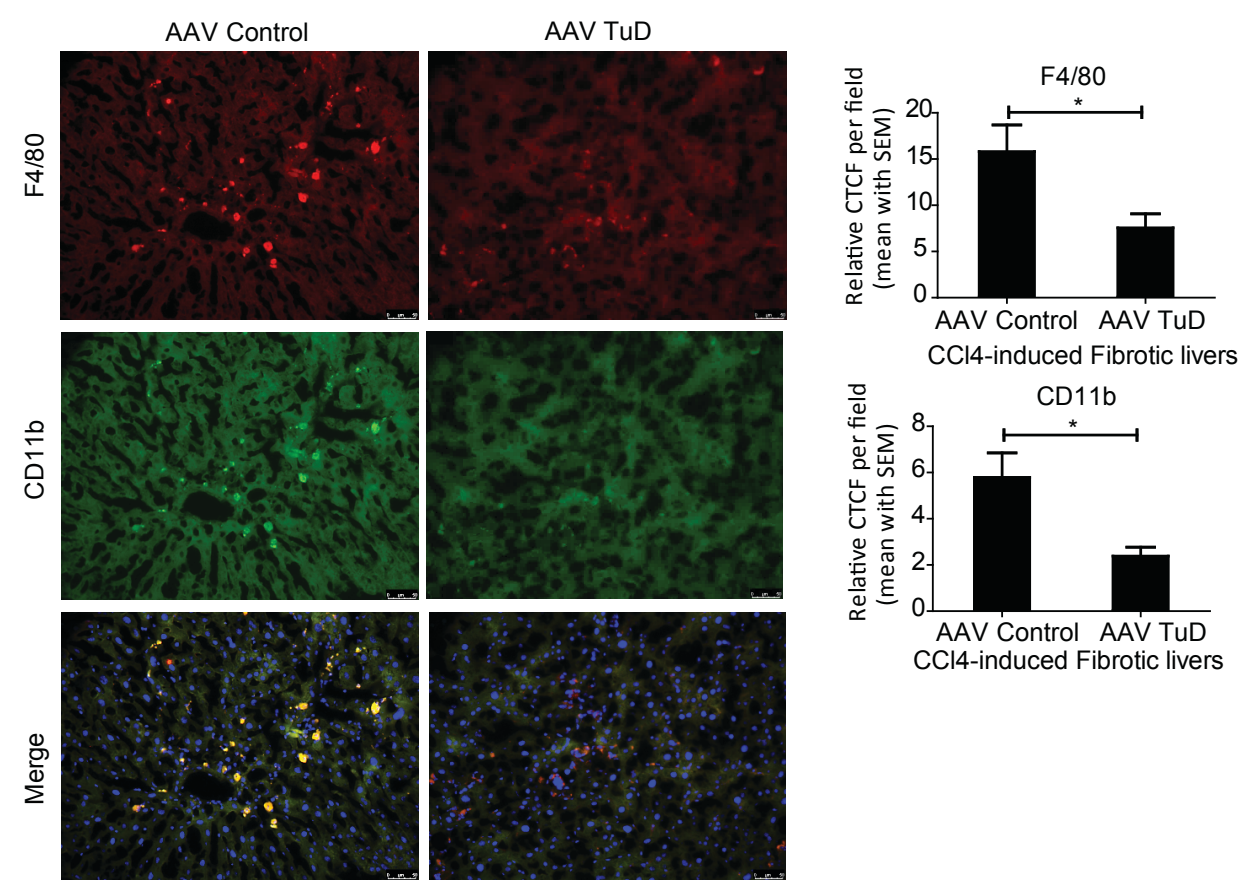

Supplementary Fig. S8. Reduced monocyte recruitment due to decreased levels of CCL2 upon miR-221-3p inhibition contributes to amelioration of liver fibrosis. (A) FACS analyses and

immunofluorescence staining for CD11b and F4/80 show reduced number of double positive cells in mice injected with AAV TuD ( $n=11$ fields/group). Error bars represent \pm SEM. One-way ANOVA was used for statistical evaluation. $\left({ }^{*} p<0.05\right)$. Depicted FACS plots in panel A are pregated on CD45.2 ${ }^{+}$, Ly6G $^{-}$ 
leucocytes.

\section{Figure S9}

A
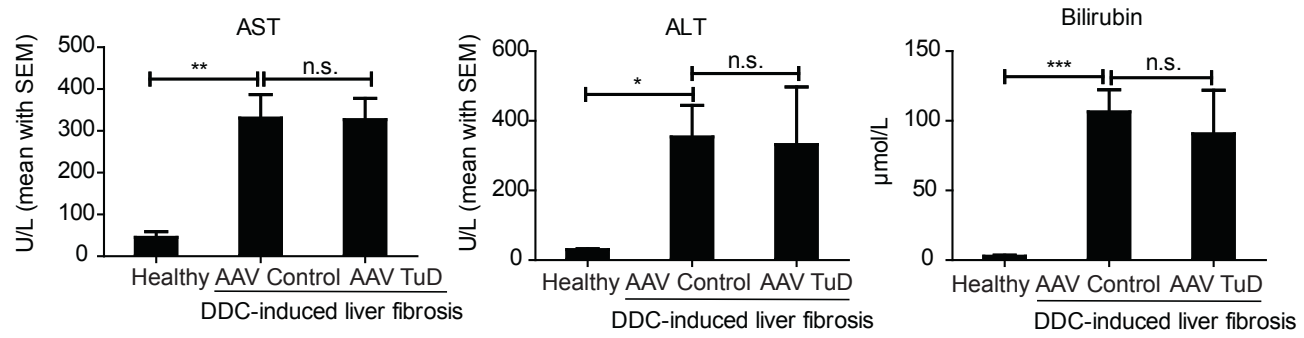

B

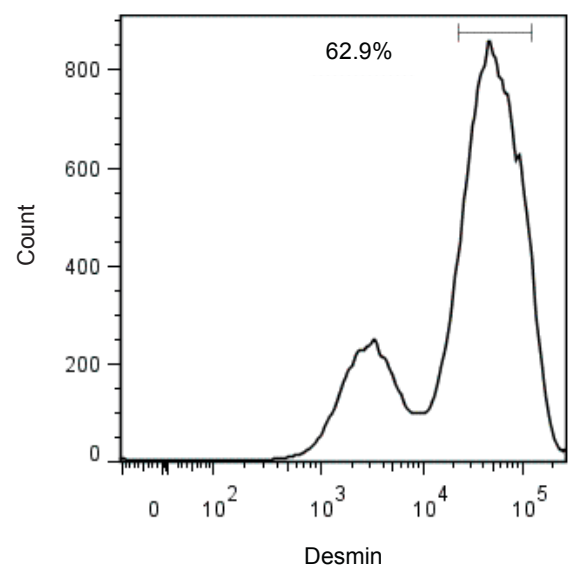

Supplementary Fig. S9. (A) Analysis of serum from mice fed with DDC

diet. No significant difference of AST, ALT, and bilirubin in serum from DDC-induced liver fibrotic mice injected either with AAV TuD ( $n=5$ mice/group or AAV Control, $n=3$ mice/group in AAV TuD). (B) FACS for desmin positive cells show $62.9 \%$ purity of HSCs. Error bars represent \pm SEM. One-way ANOVA was used for statistical evaluation. $\left({ }^{*} p<0.05 ;{ }^{* *} p<0.01 ;{ }^{* * *} p<0.001\right)$. 